\title{
Rheology and Dynamics of Associative Polymers in Shear and Extension: Theory and Experiments
}

\author{
Anubhav Tripathi, $* \dagger$ Kam C. Tam,, and Gareth H. McKinley ${ }^{\S}$ \\ Division of Engineering, Brown University, Providence, Rhode Island 02916; School of Mechanical \\ and Aerospace Engineering, Nanyang Technological University, 50 Nanyang Avenue, \\ Singapore 639798, Singapore; and Department of Mechanical Engineering, M.I.T. \\ Cambridge, Massachusetts 02139
}

Received July 22, 2005; Revised Manuscript Received November 16, 2005

\begin{abstract}
We investigate the steady and transient shear and extensional rheological properties of a series of model hydrophobically modified ethoxylate-urethane (HEUR) polymers with varying degrees of hydrophobicity. A new nonlinear two-species network model for these telechelic polymers is described which incorporates appropriate molecular mechanisms for the creation and destruction of elastically active chains. Like other recent models we incorporate the contributions of both the bridging chains (those between micelles) and the dangling chains to the final stress tensor. This gives rise to two distinct relaxation time scales: a short Rouse time for the relaxing chains and a longer network time scale that depends on the aggregation number and strength of the micellar junctions. The evolution equations for the fraction of elastically active chains and for the conformation tensors of each species are solved to obtain the total stress arising from imposed deformations. The model contains a single adjustable nonlinear parameter and incorporates the nonlinear chain extension, the shear-induced enhancement of associations, and the stretch-induced dissociation of hydrophobic chains. In contrast to earlier closed-form models, we are able to obtain quantitative agreement between experimental measurements and the model predictions for three different series of telechelic polymers over a range of concentrations. The scaling of both the zero shear viscosity and the effective network relaxation time shows good agreement with those measured in experiments. The model also quantitatively captures both the shear thickening and subsequent shear thinning observed in the rheology at high deformation rates and predicts transient extensional stress growth curves in close agreement with those measured using a filament stretching rheometer.
\end{abstract}

\section{Introduction}

Hydrophobically modified, water-soluble polymers or "associative polymers" are a class of complex and partially ordered systems that have attracted a great deal of interest in recent years. These materials are aqueous soluble block copolymers that contain both water-soluble (hydrophilic) and water-insoluble components of varying levels of hydrophobicity. As a result of the amphiphilic character of the molecules, they may act, even at low concentration, as effective rheological modifiers. They can therefore be used for various industrial applications in which careful control of the rheology of the solution is required, e.g., paints, foods, and pharmaceuticals. Examples of these triblock or telechelic polymers include hydrophobically ethoxylated urethanes (HEURs) with hydrophobic end groups consisting of aliphatic alcohols, alkylphenols or fluorocarbons, and hydrophobically modified alkali-soluble ethoxylates (HASE) with hydrophobic macromonomers distributed along the polymer backbone. The driving force for the association process is the interaction between the hydrophobic segments that arises in order to minimize their exposure to water. The resulting micellar associations give rise to both intra- and intermolecular temporary domains or junctions. The network is temporary in the sense that the junctions that hold the network together break and reform continuously due to thermal fluctuations. Numerous research groups have investigated the association and rheology of these associative polymer systems. The review by Winnik

\footnotetext{
* Corresponding author.

†rown University.

Nanyang Technological University.

$\S$ M.I.T.
}

and Yekta ${ }^{1}$ and the text of Larson ${ }^{2}$ provide a detailed survey of this literature.

In the present study we focus on the behavior of a set of model telechelic HEUR polymers. These linear triblock systems are ostensibly the simplest of the many associative polymer materials available. There have been many experimental studies of the viscoelastic properties of these aqueous solutions and also a number of theoretical models based on transient network theory that appear to capture, at least qualitatively, the experimental observations. However, there are few studies that quantitatively compare rheological measurements with constitutive predictions. This is the focus of the present work.

In a pair of recent papers, Pellens et al. ${ }^{3,4}$ identify at least five signature features of rheological nonlinearity in associative polymer networks that must be captured by any theoretical model. They also demonstrated that although these features may be captured qualitatively by existing constitutive equations, quantitative discrepancies with experimental measurements that may be of an order of magnitude or more typically persist.

Depending on the relative locations of the two hydrophobic end groups of the telechelic polymer chain, different scenarios can be visualized. ${ }^{5}$ Loops are formed if the end groups of the polymer are present in the same micelle. On the other hand, it is possible that the hydrophobic end groups are located in different micelles, and in this case, a bridge is established between two micelles. These bridging chains may be referred to as elastically active since they form part of the temporary elastic network. Depending upon the hydrophobicity of the end group and the polymer concentration, one or both ends can also temporarily exist without any association. These molecules form dangling chains which can relax before being reincorporated 
into the micellar network. The complex rheological response of telechelic polymers originates from the dynamical interchange of these loops, bridges, and dangling chains as a function of the deformation imposed on the network. The structure and dynamics of these telechelic chains depends on numerous parameters such as polymer concentration, molecular weight, hydrophobe size and characteristics, degree of hydrophobicity, temperature, and the imposed shear deformation. Hence, the relationship between structure and the rheological response of associative polymers is quite complex. ${ }^{1}$

In this discussion we have inherently adopted a polymeric network view of telechelic systems, in contrast to the colloidal particle approach adopted elsewhere ${ }^{6-10}$ The elastic chains in telechelic networks are typically quite long and are expected to play an important role in governing the rheology and dynamics of the material. However, we do incorporate essential features of colloidal models in our description of the aggregation number and hydrophobic association/dissociation rates of the flowerlike micelles that form the junction points in the temporary network.

Examples of the complex rheological behavior exhibited by these polymeric networks include the ability to form highly viscous solutions at low to moderate concentrations, a linear viscoelastic response that closely resembles that of a perfect Maxwell fluid with a single relaxation time, ${ }^{5,11,12}$ shear thickening at moderate shear rates in steady shear flow followed by marked shear thinning at high shear rate, ${ }^{13-19}$ an Arrheniuslike temperature dependence of the zero-shear rate viscosity, 5,20 and a decrease in the critical shear rate at which onset of shear thickening occurs as the association strength or concentration increases or temperature decreases. ${ }^{21}$ Recently, $\mathrm{Ng}$ et al. ${ }^{20}$ disputed the assertion of a single relaxation time suggested by Annable et al. ${ }^{5}$ and demonstrated that HEUR polymers in fact possesses a dual relaxation behavior. These authors supported their claim by performing relaxation spectra analysis and by fitting a two-mode Maxwell model to their experimental data. The short time relaxation process is ascribed to the lifetime of hydrophobic junctions, ${ }^{22}$ whereas the long time process is ascribed to the network relaxation. Recently, Berret and coworkers ${ }^{23-25}$ performed startup of steady shear flow and stress relaxation experiments to study the strain hardening and shear thinning behavior of HEUR polymers. On the basis of their results, the authors suggested breakdown or rupture of the network as possible mechanisms of shear thinning behavior. The authors also interpreted strain hardening in terms of nonlinear stretching of the elastically active chains.

Finally, it should be noted that the tensile or extensional properties of the associative polymer network remain virtually unexplored even though the extensional rheology plays an important role in many commercial applications of associative polymers such as spray formation and paint applications. Recently, Sadeghy and James ${ }^{26}$ measured the apparent extensional viscosity $\eta_{\mathrm{E}}$ using a converging channel rheometer. Tan et al. ${ }^{27}$ also measured extensional properties of HASE solutions using an opposed jet device. The complex kinematics that arise in these devices, due to distribution of strains and strain rates experienced by individual fluid elements, make it difficult to interpret the extensional data unambiguously. However, the apparent extensional viscosity was found to increase roughly exponentially with the hydrophobicity and concentration of the polymers.

Several constitutive models have been developed to describe the rheological behavior of associative polymers. In a series of papers, Tanaka and Edwards ${ }^{28-31}$ developed a temporarynetwork kinetic model for telechelic polymers, by applying ideas originally formulated by Green and Tobolsky ${ }^{32}$ and Yamamoto. ${ }^{33}$ The main mechanism of stress relaxation (and shear thinning) in these models is the rate of chain detachment from the elastically active network which is activated by the elastic force in the chain. Tanaka and Edwards assumed that the chains obey Gaussian statistics and relax rapidly into their equilibrium state through Rouse dynamics upon detachment from each network point. This theory accurately captures basic observations such as a single relaxation time. However, this theory does not explain the shear thickening phenomenon at intermediate rates, and several modifications have been offered in the literature. ${ }^{13,34,35}$ However, these modifications appear to be inadequate for explaining the shear thickening behavior observed in telechelic solutions.

Annable et al. ${ }^{5}$ presented a detailed experimental study of telechelic polymer solutions and compared the results with the predictions of Monte Carlo simulations of network topology. Retaining end-capping efficiency as a parameter, these authors showed that the concentration-dependent relaxation time scales as $\lambda \sim f\left(c \sqrt{M_{\mathrm{w}}}\right)$. Here, $c$ denotes polymer concentration and $M_{\mathrm{w}}$ denotes the molecular weight of the chain. This scaling was found to be consistent with the experimental data and quantitatively captured many of the linear viscoelastic properties of the fluids. The complex nature of the model prevents the development of a closed form model suitable for analytic or numerical evaluation or for assessing the response in other modes of deformation such as uniaxial extension. Marrucci et al. ${ }^{36}$ took finite extensibility and partial relaxation of the dangling segments into account in order to qualitatively explain the shear thickening behavior observed in telechelic polymers. These authors also put forward important scaling arguments for the dependence of rheological quantities (such as the viscosity and relaxation time) on the concentration and molecular weight of the telechelic polymer chains. Ahn and Osaki ${ }^{37}$ studied a wide range of possible shear thinning and shear thickening behavior using a network model with strain-dependent bridge formation and loss rates. Van den Brule and Hoogerbrugge ${ }^{38}$ performed Brownian dynamics simulation of a complete network of telechelic chains in shear flows and analyzed their results in terms of transient network theory. These simulations showed that even in systems with Gaussian chains and fixed association and dissociation rates, shear thickening could occur. They attributed shear thickening to incomplete relaxation of a dissociating chain and also found that its probability of reattachment to the network increased linearly with the length of the chain. Hernandez-Cifre et al. ${ }^{39}$ extended these ideas to incorporate finite extensibility of the chains and nonaffine motion of the network. Vaccaro and Marrucci ${ }^{40}$ derived a simple constitutive model inspired partially by the simulation results of Van den Brule and Hoogerbrugge. ${ }^{38}$ They formulated the evolution equations for both bridging chains and temporary dangling chains by choosing simple kinetics of detachment and reattachment processes. The resulting set of nonlinear equations predicts shear thickening at moderate shear rates followed by shear thinning at much higher shear rates. This model was also used $^{4}$ for quantitative comparison with experimental measurements of the shear rheology for two different HEUR polymers. Although the model captures all the key signature effects of nonlinearity, ${ }^{3}$ it failed to capture the characteristic range of deformation rates at which shear thickening and shear thinning were observed experimentally. The authors suggest that a nonaffine motion of the elastically active chains with a negative slip coefficient (i.e., "superaffine" deformation) might capture the experimentally observed trends. 
Although the studies described above furnish various possible mechanisms for qualitatively explaining the experimentally observed nonlinear rheological phenomena, a quantitative comparison of theoretical predictions with steady shear, oscillatory shear, and transient extensional rheology data is still lacking. In this paper, we first propose a modified nonlinear constitutive model for the telechelic associative polymers, which incorporates the most important underlying molecular mechanisms. The model incorporates earlier work ${ }^{38,39}$ in which both elastically active bridging chains (those between micelles) and temporary dangling chains contribute to the final stress tensor. In contrast to full Brownian dynamics simulations of the stochastic differential equations, ${ }^{39}$ we simplify the evolution equations by ensemble averaging. The resulting ordinary differential equations for conformations and stresses carried by the two species can be solved numerically or analytically (in the limit of small shear rates). We incorporate the finite extensibility of bridging chains and the nonlinear rate of incorporation of the dangling chains into the elastic network. We then compare the model predictions with a systematic experimental study of the linear viscoelastic, steady shear, and transient extensional properties of a series of well-characterized model hydrophobically modified ethoxylate-urethane (HEUR) polymers possessing varying degrees of hydrophobicity.

\section{Model Development}

2.1. Molecular Architecture. We seek to understand the behavior of associative polymers in aqueous solution by studying model polymers of well-defined structures. Consider a hydrophilic flexible chain of molecular weight $M_{\mathrm{w}}$ consisting of $N$ statistically independent segments each of length $b$ (the "Kuhn length"). The number of segments and the "Kuhn length" in the equivalent freely jointed Kuhn chain are computed as

$$
N=\frac{3 M_{\mathrm{w}} \sin ^{2} \theta}{M_{0} C_{\infty}} \text { and } b=\frac{C_{\infty} l}{\sin \theta}
$$

where $C_{\infty}$ (= 4 for poly(ethylene oxide) chains) is the characteristic ratio, $l(=0.154 \mathrm{~nm})$ is the carbon-carbon bond length, $M_{0}(=44 \mathrm{~g} / \mathrm{mol})$ is the molecular mass of the repeat unit, and $\theta\left(=54.5^{\circ}\right)$ is the half-angle between carbon-carbon bonds in a polymer chain. The root-mean-square end-to-end distance of the equivalent Kuhn length is $\left\langle R^{2}\right\rangle^{1 / 2}=\sqrt{N b^{2}}$. The hydrophilic chain is also end-capped with small chains of $n_{\mathrm{c}}$ $\left(n_{\mathrm{c}} \ll N\right)$ hydrophobic units. This leads to so-called telechelic polymers. We then study an aqueous solution of such polymer chains of narrow molecular weight distribution with a concentration $c$ per unit volume. The number density of the chains is computed as $n=c N_{\mathrm{A}} / M_{\mathrm{w}}$, where $N_{\mathrm{A}}$ is Avogadro's number. The synthesis procedure and characterization of the polydispersity of the molecules are described elsewhere. ${ }^{12,27}$

Research to date has established that such polymers form micelles in dilute solutions above a rather low critical micelle concentration $\left(c_{\mathrm{cmc}} \ll 0.1 \mathrm{wt} \%\right)^{12}$ which depends on the backbone molecular weight $M_{\mathrm{w}}$ and the hydrophobe length $n_{\mathrm{c}}$. The telechelic polymers undergo simultaneous formation of intramolecular micelles with a dense core of hydrophobic groups and interchain micellar cross-links. Thermodynamic studies have shown that at moderately dilute concentrations perfectly endcapped telechelic polymers may also phase separate ${ }^{7-9}$ into a viscous gel phase and an aqueous phase. This tendency for phase separation is suppressed by further increases in concentration so that the elastically active PEO chains overlap and also by imperfect end-capping of the telechelic polymers. ${ }^{1}$ In the present study we focus on sufficiently high concentrations and molecular weights that no phase separation is observed. Thus, depending upon the thermodynamics of the association process, a single phase solution of telechelic chains contains several types of possible chain structures: "bridges", "loops", "danglers", and "free chains". A chain connecting two different micelles is called a "bridge" chain. "Loops" are formed if the end groups of the polymer are present in the same micelle. Similarly, "danglers" or "free chains" are formed when one or both ends temporarily exist without any association. At low concentrations, the loops dominate, but with an increase in the polymer concentration, the number of micelles interconnected through bridging chains increases rapidly. We specifically focus our attention in the nonentangled regime in which the number of monomers, $N$, in a polymer chain is smaller than the entanglement number, $N_{\mathrm{e}}$, i.e., the number of monomers required for the chains to become entangled. Annable and co-workers ${ }^{5}$ also discuss the possible existence of shear-induced creation of more complex multiplybridged structures; however, these are beyond the scope of the present work.

In aqueous solutions, the hydrophobic ends associate into micelles that form junction points in a polymeric network. The depth of the free energy well $\Delta G$, characterizing the junction points, depends on a number of molecular parameters such as the aggregation number ${ }^{10} N_{\text {agg, }}$, the hydrophobe length as measured by $n_{\mathrm{c}}$ (the number of $-\mathrm{CH}_{2}-$ moieties ${ }^{15}$ ), the length of the polymer chain $M_{\mathrm{w}}$, and the solvent quality. ${ }^{8}$ Owing to ambient thermal noise $\left(\sim k_{\mathrm{B}} T\right)$, there is a finite probability that a micellar hydrophobe acquires sufficient energy to overcome the activation barrier $\Delta G$ and detach spontaneously. Following the model of Bell ${ }^{41}$ and Tanaka and Edwards, ${ }^{31}$ the exit rate, $1 / \tau_{\mathrm{E}}$, is estimated as the product of a natural thermal vibration frequency, $\Omega\left(\sim 10^{8}-10^{10} \mathrm{~Hz}\right)$, of the hydrophobic association in a micelle and the quasi-equilibrium likelihood of reaching a transition state with an energy barrier $\exp \left(-\Delta G / k_{\mathrm{B}} T\right)$. Hence, we expect

$$
\frac{1}{\tau_{E}}=\Omega \exp \left(-\frac{\Delta G}{k_{\mathrm{B}} T}\right)
$$

where the association energy is estimated to be $\sim 0.98 k_{\mathrm{B}} T$ per $-\mathrm{CH}_{2}-$ unit for an alkane hydrophobe ${ }^{5,8}$ so that $\Delta G \approx 0.98 n_{\mathrm{c}} k_{\mathrm{B}} T$. Theoretically, the thermal vibration frequency is expected to be inversely proportional to the local friction constant for micellar motion in the viscous solvent. For $\Delta G / k_{\mathrm{B}} T \gg 1$, it is increasing unlikely for hydrophobes to exist outside the micellar core, and the solution is devoid of any completely unassociated free chains or permanent danglers. Hence, for associative polymer solutions, with hydrophobic end groups of 10 or more methylene units, bridges and temporarily ejected chains with single dangling ends are likely to carry most of stress in the solutions. The number of elastically active chains $v$ (i.e., those in aggregates) is then defined ${ }^{31}$ as

$$
v=n\left(\frac{\mathrm{e}^{\Delta G / k_{\mathrm{B}} T}}{1+\mathrm{e}^{\Delta G / k_{\mathrm{B}} T}}\right)
$$

with $v \cong n$ for $\Delta G / k_{\mathrm{B}} T \gg 1$.

The aggregate number of hydrophobic units $N_{\text {agg }}$ contained in a single micelle depends on the molecular structure of the hydrophobe. More specifically, it is related to the volume of each hydrophobic moiety and the area of the micelle surface required to accommodate each hydrophobe within the micelle. Using fluorescence decay studies, Yekta and co-workers ${ }^{15,16}$ have deduced a micelle aggregation number of $N_{\mathrm{agg}} \approx 18-28$ 
hydrophobes per micelle. Fluorescence studies detect no change in micellar size from dilute concentrations up to $7 \mathrm{wt} \%$. This supports the notion that increasing the concentration above the critical micelle concentration $c_{\mathrm{cmc}}$ primarily increases the number of micelles at a fixed value of $N_{\mathrm{agg}}$, much as for low molecular weight surfactant systems. As the number of micelles increases, the average distance between aggregates, denoted as $\langle a\rangle$, decreases. Since, on average, a single aggregate/micelle occupies a volume of $4 \pi\langle a\rangle^{3} / 3$, the average spatial distance $\langle a\rangle$ is related to the number of active chains $n=c N_{\mathrm{A}} / M_{\mathrm{w}}$, of which carries two hydrophobic ends by the expression

$$
\langle a\rangle=\left(\frac{3 N_{\mathrm{agg}}}{8 \pi n}\right)^{1 / 3}
$$

This lattice-based definition relates physical properties of the individual polymer to the resulting micellar solution structure. Marrucci et al. ${ }^{36}$ adopted the lattice approach to estimate the effect of partial relaxation of the detached chains. These authors argued that the mean distance traveled by a detached hydrophobe depends on the ratio of the radius of gyration of the chain $l_{\mathrm{c}}=\sqrt{N b^{2} / 3}$ and the lattice spacing $\langle a\rangle$. Hence, if $\langle a\rangle^{2}<l_{\mathrm{c}}^{2}$, a detached chain generally rejoins the network before it can completely relax its original deformation, and the "effective" relaxation time of the chain, due to presence of a partially deformed network, becomes

$$
\tau_{\text {eff }} \sim \frac{N b^{2} / 3}{\langle a\rangle^{2}} \tau_{E}
$$

Substituting $N \sim M_{\mathrm{w}}$ and $\langle a\rangle^{2} \sim\left(M_{\mathrm{w}} / c\right)^{2 / 3}$, we find that the effective relaxation time scales nonlinearly with both concentration and the molecular weight as $\tau_{\text {eff }} \sim\left(c \sqrt{M_{\mathrm{w}}}\right)^{2 / 3}$. This result is consistent with the experimental findings of Annable. ${ }^{5}$ Since $\tau_{\text {eff }}$ is the relaxation time of the network, the zero-shear viscosity can be expressed as ${ }^{32}$

$$
\eta_{0} \cong G_{N}^{0} \tau_{\mathrm{eff}} \cong n k_{\mathrm{B}} T \tau_{\mathrm{eff}}
$$

where $G_{N}^{0}$ is the plateau modulus of the physical network. From eq 6 we then find the zero-shear-rate viscosity is proportional to $c^{5 / 3} M_{\mathrm{w}}{ }^{-2 / 3}$. Consideration of the lattice spacing thus predicts an augmented dependence on concentration, which is in good agreement with the experimental findings of Annable et al. ${ }^{5}$

2.2. Constitutive Equation. Following Van den Brule and Hoogerbrugge, ${ }^{38}$ we focus on two species of chains (elastically active polymer chains) which carry most of the stress in a telechelic polymer system: the bridges and the temporarily dangling chains with single dangling ends. The looped chains contribute to the stress only inasmuch as they are polymer molecules of radius of gyration $\sqrt{\left\langle R_{\mathrm{g}}{ }^{2}\right\rangle}=\sqrt{N b^{2}}$ which can be deformed by the flow. Although our model can easily be extended to account explicitly for the exchange between loops and dangling chains, at this stage, we assume that the loops play no additional role in the total stress tensor. The telechelic polymer solution is assumed to remain in a single phase and the network junctions (i.e., the micelles) move affinely. Each telechelic polymer molecule is modeled as a Gaussian chain, and we take the chains to be freely jointed, bead-spring chains, ${ }^{42}$ in which the end-to-end distance is represented generically by a vector $\underline{Q}$. Because we consider two species, when considering bridging chains, we explicitly denote the endto-end vector $\underline{Q}_{\mathrm{A}}$ and for dangling chains we use the notation
$Q_{\mathrm{B}}$. To specify the orientation of the bridge, we introduce the distribution functions $\Psi_{\mathrm{A}}(\underline{Q}, t)$, normalized to the number of bridge chains per unit volume $v_{\mathrm{A}}$, as $v_{\mathrm{A}} \psi_{\mathrm{A}}(\underline{Q}, t)$ and the distribution function $\Psi_{\mathrm{B}}(\underline{Q}, t)$ for the temporarily detached dangling chains, normalized as $\left(v-v_{\mathrm{A}}\right) \psi_{\mathrm{B}}(\underline{Q}, t)$ with $v$ the total number of elastically active chains per unit volume. Hence, the distribution functions $\psi_{\mathrm{A}}, \psi_{\mathrm{B}}$ satisfy $\int \psi_{\mathrm{A}} \mathrm{d} V=\int \psi_{\mathrm{B}} \mathrm{d} V=1$, where $\mathrm{d} V$ is a volume element in configuration space.

Following standard methods, ${ }^{38,42}$ we then perform balances in configuration space for the evolution of the distribution function for the bridging chains to obtain

$$
\frac{\partial \Psi_{\mathrm{A}}}{\partial t}=-\frac{\partial}{\partial \underline{Q}} \cdot\left[\kappa \cdot \underline{Q} \Psi_{\mathrm{A}}\right]+L(\underline{Q}, t) \Psi_{\mathrm{B}}-M(\underline{Q}, t) \Psi_{\mathrm{A}}
$$

where $\kappa=\nabla \mathrm{v}^{\mathrm{T}}$ denotes the transpose of the velocity gradient tensor, $L(Q, \bar{t})$ denotes the probability rate of creation of the bridging chains of length $Q_{\mathrm{A}}$, and $M(Q, t)$ denotes the probability rate of destruction of the bridging chains of length $\underline{Q}_{\mathrm{A}}$. Equation 7 assumes affinely deforming bridging chains although it is straightforward to incorporate nonaffine motion. ${ }^{4}$ Similarly, we can write the convection equation ${ }^{38}$ that determines the distribution function of the temporally ejected dangling chains as

$$
\begin{aligned}
\frac{\partial \Psi_{\mathrm{B}}}{\partial t}=-\frac{\partial}{\partial \underline{Q}} \cdot\left[\kappa \cdot \underline{Q} \Psi_{\mathrm{B}}-\frac{k_{\mathrm{B}} T}{\zeta} \frac{\partial}{\partial \underline{Q}} \ln \Psi_{\mathrm{B}}-\frac{1}{\xi} F^{(\mathrm{c})}\right]- \\
L(\underline{Q}, t) \Psi_{\mathrm{B}}+M(\underline{Q}, t) \Psi_{\mathrm{A}}
\end{aligned}
$$

where $\zeta$ is the friction coefficient and $F^{(\mathrm{c})}$ is the spring or connector force. The two additional terms on the right-hand side represent contributions due to thermal diffusion of free hydrophobic ends through the solvent and the elasticity of the connecting chain. Once the chain is free at one end, it can relax its stress through its Rouse modes. Hence, eq 8 describes nonaffine motion of temporarily dangling chain. Following dumbbell theory, ${ }^{42}$ we write the connector force law as

$$
\underline{F}^{(\mathrm{c})}=H \underline{Q f}(\underline{Q})
$$

where $H=3 k_{\mathrm{B}} T / N b^{2}$ is a spring constant and $f \equiv 1 /\left(1-Q^{2} /\right.$ $Q_{\max ^{2}}{ }^{2}$ is the nonlinear Warner spring factor accounting for the finite extensibility of the polymer chain. Finally, for a given deformation tensor $k$, we need to solve eqs $7-9$ for $\Psi_{\mathrm{A}}(\underline{Q}, t)$ and $\Psi_{\mathrm{B}}(\underline{Q}, t)$ and then calculate the resulting stress field $\bar{\sigma}$ as

$$
\boldsymbol{\sigma}=v_{\mathrm{A}}\left\langle\underline{F}_{\mathrm{A}}{ }^{(\mathrm{c})} \underline{Q}_{\mathrm{A}}\right\rangle+\left(v-v_{\mathrm{A}}\right)\left\langle\underline{F}_{\mathrm{B}}{ }^{(\mathrm{c})} \underline{Q}_{\mathrm{B}}\right\rangle
$$

where the angle brackets denote ensemble averages over conformation space with respect to the corresponding distribution functions. Note that we have neglected terms accounting for the looped chains and the solvent contribution to the total stress.

To compute the stress field $\boldsymbol{\sigma}$ as a function of a given deformation field $\kappa+\kappa^{\mathrm{T}}$, the number density of bridging polymers, $v_{\mathrm{A}}$, and the distribution functions $\psi_{\mathrm{A}}(Q, t)$ and $\psi_{\mathrm{B}}(\underline{Q}, t)$ need to be obtained from the coupled nonlinear partial differential eqs 7 and 8 . We also need to specify the exact forms of $L(Q, t)$ (i.e., the probability rate of creation of the bridging chains of length $Q_{\mathrm{A}}$ and $M(Q, t)$ (i.e., the probability rate of destruction of the bridging chains). Although the above set of equations can be converted to the equivalent stochastic differential equation and then solved directly using techniques such as Brownian dynamics, ${ }^{38,43}$ we seek to develop a simple closedform model by making suitable approximations without losing too much of the essential physics. Hence, we replace $\underline{Q}$ by the 
Table 1. Comparison of Creation and Destruction Rate for Various Models ${ }^{a}$

\begin{tabular}{lcl}
\hline \multicolumn{1}{c}{ model } & probability rate of creation $L(\underline{Q}, t)$ & probability rate of destruction $M(\underline{Q}, t)$ \\
\hline Green and Tobolsky ${ }^{32}$ & $1 / \tau_{\mathrm{E}}$ & $1 / \tau_{\mathrm{E}}$ \\
Tanaka and Edwards ${ }^{28-31}$ & $c_{1} / \tau_{\mathrm{E}}$ & $\exp \left(c_{2} Q\right) / \tau_{\mathrm{E}}$ \\
& & $\left(c_{3}+1.5 c_{4} Q^{2}\right) / \tau_{\mathrm{E}}$ \\
Wang $^{34}$ & $\left(c_{1}+c_{2} \tau_{\mathrm{E}}{ }^{2} \dot{\gamma}^{2}\right) / \tau_{\mathrm{E}}$ & $\left(1+c_{3} Q^{2} / Q_{\mathrm{eq}}\right) / \tau_{\mathrm{E}}$ \\
Ahn and Osaki & $\exp \left(c_{1} \gamma\right) / \tau_{\mathrm{E}}$ & $\exp \left(c_{2} \gamma\right) / \tau_{\mathrm{E}}$ \\
van den Brule and Hoogerbrugge $^{38}$ & $\left(c_{1} Q / Q_{\mathrm{eq}}\right) / \tau_{\mathrm{E}}$ & $2 / \tau_{\mathrm{E}}$ \\
Hatzikiriakos and Vlassopoulos & \\
Vaccaro and Marrucci ${ }^{40}$ & $2 Q^{3} n_{1} \dot{\gamma} / 3$ & $4 D / Q^{2} \exp \left(-W / k_{\mathrm{B}} T\right)$ \\
Hernandez-Cifre et al. ${ }^{39}$ & $\left(c_{1}+c_{2} Q / Q_{\mathrm{eq}}\right) / \tau_{\mathrm{E}}$ & $f / \tau_{\mathrm{E}}$ \\
& $1-\exp \left\{-\left(c_{1}+c_{2} f Q\right) \Delta t\right\}$ & $1-\exp \left\{-2 \Delta t \exp \left(c_{3} f^{2}\right) / \tau_{\mathrm{E}}\right\}$
\end{tabular}

${ }^{a}$ Here $c_{1}, c_{2}, c_{3}$, and $c_{4}$ are model specific constants; $\gamma$ is the total strain; $\dot{\gamma}$ is the shear rate; $f$ is given in eq 9 ; $n_{1}$ is the concentration of single molecules; $D$ is the translational diffusivity of a molecule; $W$ is the energy of interaction of two molecules; $Q=\sqrt{\left\langle Q^{2}\right\rangle}$ is the ensemble average length of the bridged chain; $\Delta t$ is the simulation time step.

preaveraged quantity $Q=\sqrt{\left\langle\underline{Q}^{2}\right\rangle}$ in (i) the expression for finite extensibility factor ${ }^{44,45} f$, (ii) the expression for probability rate of creation of the bridging chains $L(Q, t)$, and (iii) the probability rate of destruction of the bridging chains $M(Q, t)$. Therefore, at any instant, the above terms are taken to be independent of the distribution $\underline{Q}$ but dependent on the ensemble average of $Q^{2}$. Recent studies ${ }^{46,47}$ have shown that preaveraging can be inaccurate in strong flows; however, for steady-state flows, preaveraging seems to be an acceptable approximation. ${ }^{44,48}$

Before integrating the evolution equations over configuration space, we introduce the dimensionless conformation tensors A for the bridging chains and $\mathbf{B}$ for the temporary dangling chains, which are computed by averaging the approximate dyadic product $\underline{Q Q}$ over the respective conformation distribution spaces to give

$$
\mathbf{A}=\frac{1}{l_{\mathrm{c}}^{2}} \int \underline{Q}_{\mathrm{A}} \underline{Q}_{\mathrm{A}} \psi_{\mathrm{A}} \mathrm{d} V \text { and } \mathbf{B}=\frac{1}{l_{\mathrm{c}}^{2}} \int \underline{Q}_{\mathrm{B}} \underline{Q}_{\mathrm{B}} \psi_{\mathrm{B}} \mathrm{d} V
$$

where $l_{\mathrm{c}}=\sqrt{N b^{2} / 3}$ is the characteristic length for scaling such that $\operatorname{tr} \mathbf{A}_{\mathrm{eq}}=\operatorname{tr} \mathbf{B}_{\mathrm{eq}}=3$.

To obtain evolution equations for the orientation tensors describing the bridges and dangling chains $\mathbf{A}$ and $\mathbf{B}$, we multiply eqs 7 and 8 by $\underline{Q Q}$ and integrate over the configuration space. This leads to

$$
(\phi \mathbf{A})_{(1)}=(1-\phi) \bar{L}(Q, t) \mathbf{B}-\phi \bar{M}(Q, t) \mathbf{A}
$$

and

$$
\begin{aligned}
((1-\phi) \mathbf{B})_{(1)}=\frac{\tau_{\mathrm{E}}}{2 \tau_{\mathrm{R}}}(1-\phi) \boldsymbol{\delta}-\frac{\tau_{\mathrm{E}}}{2 \tau_{\mathrm{R}}} f(Q) \mathbf{B}- \\
(1-\phi) \bar{L}(Q, t) \mathbf{B}+\phi \bar{M}(Q, t) \mathbf{A}
\end{aligned}
$$

where ()$_{(1)}$ denotes the upper convected derivative, which is defined as

$$
()_{(1)}=\frac{D}{D t}()-\nabla_{\underline{\mathrm{v}}}^{\mathrm{T}} \cdot(\mathrm{)})\left(\mathrm{)} \cdot \nabla_{\underline{\mathrm{v}}}\right.
$$

The dimensionless fraction of bridged chains is denoted $\phi=$ $\nu_{\mathrm{A}} / v ; \tau_{\mathrm{R}}=\xi / 4 H$ denotes the relaxation time of free chains, and $\bar{L}$ and $\bar{M}$ are dimensionless creation and destruction rates. Note that the hydrophobe exit time $\tau_{\mathrm{E}}$ given by eq 2 has been used to nondimensionalize both time and the rate of creation and destruction. To compute the stress tensor, given by eq 10, we also need an evolution equation for the number of bridging chains per unit volume. Hence, we integrate eq 7 over all configurations to obtain

$$
\frac{D \phi}{D t}=(1-\phi) \bar{L}(Q, t)-\phi \bar{M}(Q, t)
$$

Finally, we write the stress calculator by the FENE force law described by eq 10 as

$$
\boldsymbol{\sigma}=\phi f\left(Q_{\mathrm{A}}\right) \mathbf{A}+(1-\phi) f\left(Q_{\mathrm{B}}\right) \mathbf{B}
$$

where the stress is made dimensionless using the plateau modulus $G_{N}^{0}=v k_{\mathrm{B}} T$.

The constitutive model is represented by the set of eqs $12-$ 16 in which we compute the stress field $\boldsymbol{\sigma}$, the fraction of bridging chains $\phi$, and the orientation tensors $\mathbf{A}$ and $\mathbf{B}$, for a given deformation field $\kappa+\kappa^{\mathrm{T}}$. It still remains to model the probability of creation and destruction of bridging chains in a telechelic polymer solution.

It is nontrivial to correctly formulate the creation and destruction processes, and numerous authors have proposed suitable models. Table 1 summarizes some of these attempts. Green and Tobolsky ${ }^{32}$ initially took the rate of creation and the rate destruction to be constant and their model reduces to the upper convected Maxwell model. ${ }^{42}$ Tanaka and Edwards ${ }^{28-31}$ introduced a chain-length-dependent dissociation rate to predict the rheological properties of the physically cross-linked networks. Tanaka and Edwards estimated the force required to pull a hydrophobe from a micellar well and showed that the detachment rate increases exponentially with the force acting on the polymer chain. In addition to the bridging and the dangling chains, Wang ${ }^{34}$ also considered the presence of free chains. He rationalized the shear thickening behavior by proposing a quadratic shear rate dependence of the recombination of free chains. This model also postulated a weak algebraic dependence of destruction rate on chain end-to-end vector. Ahn and Osaki ${ }^{37}$ introduced phenomenological expressions for the microscopic creation and destruction rates in terms of the effective macroscopic strain $\gamma=\left(\tau_{11}-\tau_{22}\right) / 2 \tau_{12}$ and enumerated 16 different rheological scenarios. Van den Brule and Hoogerbrugge $^{38}$ found from Brownian dynamics simulations that the probability of reattachment increases linearly with the length of chain. As a result of this mechanism, the fraction of long chain segments present in the network can increase with imposed flow, and this can explain the shear thickening observed experimentally. Hatzikiriakos and Vlassopoulos ${ }^{49}$ also performed Brownian dynamics simulations of shear-induced thickening of dilute polymer solutions. Although this work primarily considered shear thickening of nonmicellar polymer solutions, the model is of the same basic form and considered the dynamical evolution of two species with nonlinear creation and destruction rates given by the expressions in Table 1. Recently, Vaccaro 


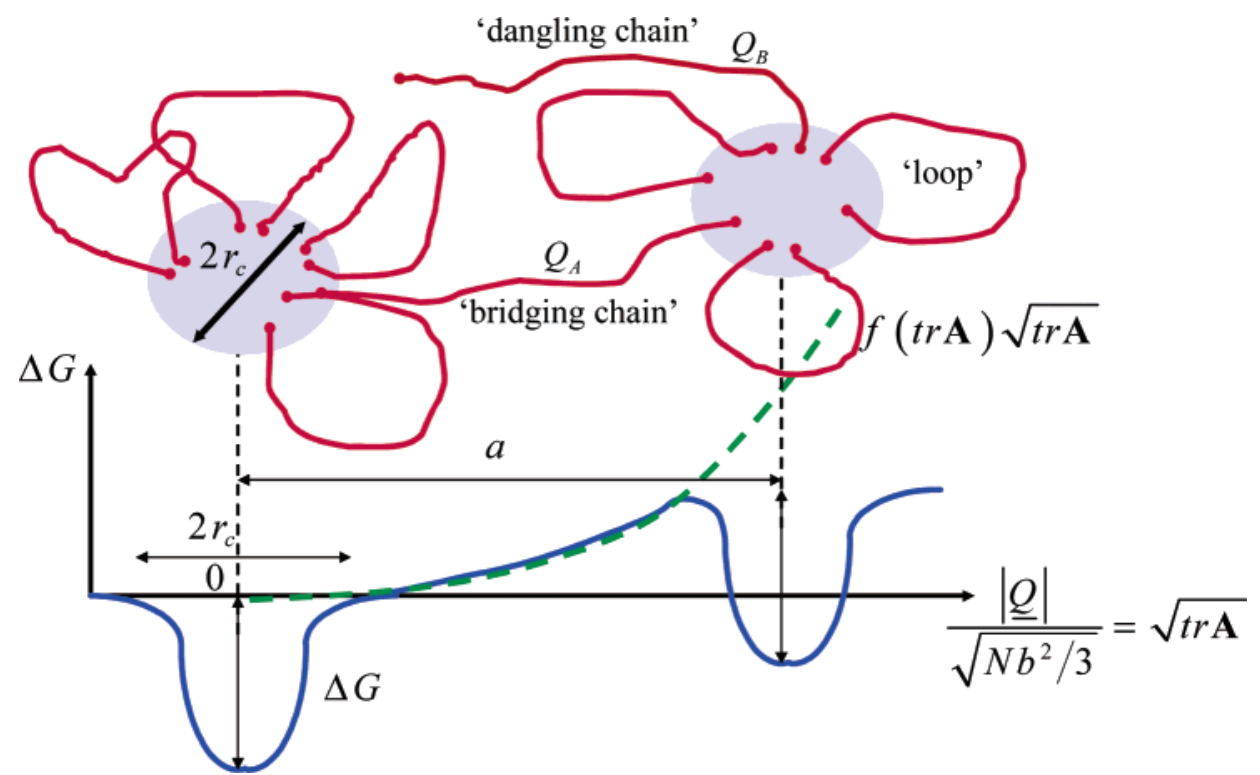

Figure 1. Conceptual view of the energy landscape for the detachment of a hydrophobe in extension. When a deformation is applied to the system, the free energy difference $\Delta G$ between the native and detached state is skewed by work performed by the tensile force on the chain.

and Marrucci $^{40}$ have developed a simple closed-form model for the nonlinear rheology of associative polymers based on the van den Brule and Hoogerbrugge study. In this model, the kinetic rate of creation is proportional to the chain length, and the rate of destruction is proportional to thermal fluctuations of the hydrophobes.

All of the above studies have provided important insights toward understanding the complex molecular processes of association and dissociation for elastically active chains; however, they all lack quantitative comparison with experimental measurements of the rheological properties of associative polymers in steady shear and/or small-amplitude oscillatory shear flows. Extensive attempts in our laboratory to fit measured viscometric data to any of the existing models have resulted in less than quantitative agreement. Similar findings are reported very recently by Pellens et al. ${ }^{4}$ We have thus returned to the basic formulation of Green and Tobolsky and Tanaka and Edwards. By appropriately incorporating each of the features elucidated in the Brownian dynamics studies, we seek to develop a closed-form constitutive model that can quantitatively describe the rheology of HEUR solutions. In the following two sections, we attempt to understand the processes of dissociation and creation of active chains by considering relevant the thermodynamically driven mechanisms and their relation to deformation-induced structural changes in the elastic network.

2.3. Probability Rate of Destruction of Active Chain. In a quiescent solution, the hydrophobic ends located in micellar aggregates experience ambient thermal fluctuations $\left(\sim k_{\mathrm{B}} T\right)$. Such fluctuations occasionally induce sufficient energy in a micellar hydrophobe to allow it to overcome the large activation barrier $\Delta G$ separating the micelle from the solvent. This thermodynamic activity establishes an overall rate of dissociation $1 / \tau_{\mathrm{E}}$, given by eq 2 , of hydrophobes in a solution containing telechelic polymers. The equilibrium thermodynamically driven probability rate of dissociation is independent of the end-to-end distance of the chain. (In fact, Green and Tobolsky ${ }^{32}$ first considered this simplest model for $M(\underline{Q}, t)$.)

Under an external deformation field, the bridging polymer chains connecting the micellar hydrophobes are stretched and impart a net force on a hydrophobhic end group residing in the micelle. If the chains are very long, or the concentration is high, then this force can be described by a linear spring; however, for large deformations the force is better described by the FENE expression of Warner ${ }^{42}$ given by eq 9. Following HernandezCifre et al., ${ }^{39}$ we incorporate the effect of this nonlinear spring force on the exit rate of the hydrophobes from the micellar junctions. As indicated in Figure 1, the tensile force in the chain distorts the energy well by an amount that is proportional ${ }^{50}$ to $\int_{Q_{\mathrm{A}}-r_{\mathrm{c}}}^{Q^{(\mathrm{c})} \cdot \mathrm{d} r}$, where $r_{\mathrm{c}}$ is the displacement associated with the energy barrier. This distortion lowers the activation barrier relative to $k_{\mathrm{B}} T$ at $\left|r_{\mathrm{c}}\right|$, increases the probability of barrier crossing, and thereby increases the frequency of hydrophobe detachment. Hence, the destruction rate $M(Q, t)$, i.e., the likelihood of a hydrophobe jumping out of the energy well, can be approximated by

$$
\begin{array}{r}
M(Q, t)=g\left(c, M_{\mathrm{w}}\right) \Omega \exp \left(-\frac{1}{k_{\mathrm{B}} T}\left\{\Delta G-\int_{Q_{A}}^{Q_{\mathrm{A}}-r_{\mathrm{c}}} F^{(\mathrm{c})} \cdot \mathrm{d} r\right\}\right)= \\
\frac{g\left(c, M_{\mathrm{w}}\right)}{\tau_{\mathrm{E}}} \exp \left(\frac{3}{N b^{2}} \int_{Q_{\mathrm{A}}}^{Q_{\mathrm{A}}-r_{\mathrm{c}}} f(r) r \mathrm{~d} r\right)
\end{array}
$$

where the front factor $g\left(c, M_{\mathrm{w}}\right)$ is a monotonic function of polymer concentration and molecular weight. We notice that the exit rate of the active chains or bridges is enhanced exponentially due to the deformation. The width of the activation barrier $r_{\mathrm{c}}$ depends on the attraction energy between the hydrophobes in the core of the micelle, the aggregation number, the solvent quality, and the length of hydrophobes. Semenov and co-workers ${ }^{10}$ investigated the properties of an isolated micelle in the limit of high aggregation numbers by adopting the model of Daoud and Cotton ${ }^{51}$ for triblock copolymer brushes in a good solvent. Following this study, we estimate the dimensionless width of the activation barrier as

$$
\frac{r_{\mathrm{c}}}{l_{\mathrm{c}}}=\beta\left(\frac{N}{2}\right)^{v} N_{\mathrm{agg}}^{(1-v) / 2} \sqrt{\frac{3}{N}}
$$

where $v$ is the Flory exponent for a good solvent $(=0.588)$ and $\beta$ is a numerical constant of order unity. If we substitute for the length of an individual chain in terms of the ensemble average and nondimensionalize with the characteristic length scale $l_{\mathrm{c}}=\sqrt{\mathrm{Nb}^{2} / 3}$, we finally obtain the following expression for the destruction rate from integrating the exponential term 


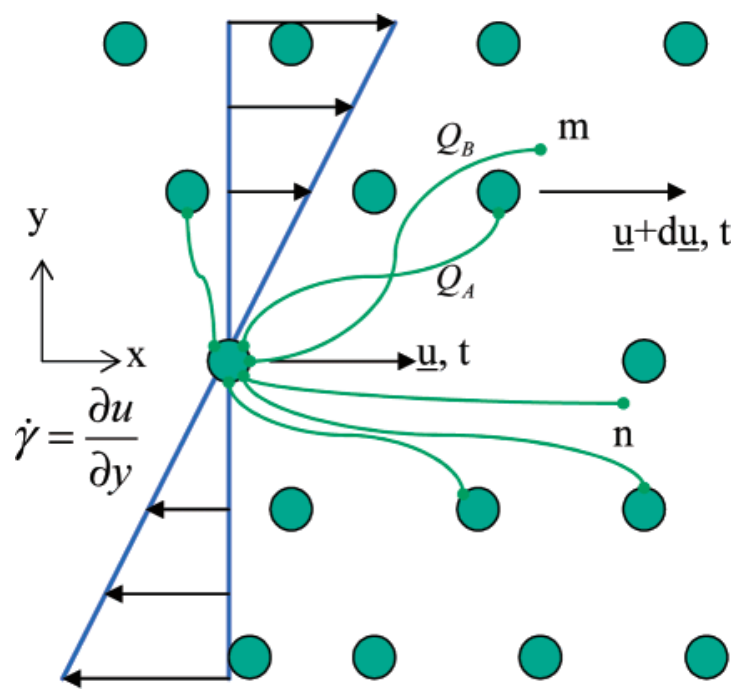

Figure 2. Schematic of two hydrophobic ends " $m$ " and " $n$ " in a lattice of micelles undergoing shear. The " $\mathrm{m}$ " end is likely to experience more interactions with neighboring micelles compared to the " $n$ " end due to the differential velocity.

in eq 19 with the Warner spring function:

$$
\bar{M}\left(Q_{\mathrm{A}}, t\right)=\bar{g}\left(c, M_{\mathrm{w}}\right)\left[\frac{1-\frac{\left(Q_{\mathrm{A}}-r_{\mathrm{c}}\right)^{2}}{3 N}}{\left(1-\frac{Q_{\mathrm{A}}^{2}}{3 N}\right)}\right]^{3 N / 2}
$$

Here $\bar{M}$ is made dimensionless using the exit time scale $\tau_{\mathrm{E}}$. The precise form of the front factor $\bar{g}\left(c, M_{\mathrm{w}}\right)$ is presented in the Results section.

2.4. Probability Rate of Creation of Active Chain. Here we propose a mechanism for the creation of active junctions which provides some insights with respect to configurational changes of the micellar-network when subjected to shear or extensional deformation and thus to the molecular origins of deformation-induced structural changes. Many diverse rheological phenomena are related to shear-induced modification of the creation and loss rates of transient molecular structure and arise from modifications to the energetic barriers for creation and destruction. Examples observed in complex fluids include assembly of $\lambda$-phage DNA, ${ }^{52}$ inhomogeneous structure formation and shear-thickening in wormlike micellar solutions, ${ }^{53}$ and multilamellar vesicle ("onions") formation in diblock copolymers. ${ }^{54}$

In the quiescent medium, thermal fluctuations bring about the transfer of ejected looped and bridged hydrophobes between the micelles. Hence, the probability rate of creation of active chains is proportional to the ejection rate $1 / \tau_{\mathrm{E}}$ of the hydrophobic ends. However, under shear or extensional deformation the association processes are expected to become considerably more pronounced owing to the strongly attracting end groups in telechelic polymers. ${ }^{34,37,38,40,49}$ The association of telechelic molecules originates from the enhanced probability that the hydrophobic end will collide with another micellar aggregate in the presence of an imposed deformation. Figure 2 shows a schematic of two hydrophobic ends " $m$ " and " $n$ " in a lattice of micelles undergoing shear. The " $\mathrm{m}$ " end is likely to experience more interactions per unit time with neighboring micelles compared to the " $n$ " end due to the differential velocity. Note that the " $m$ " and " $n$ " chains have the same length but different orientation. Hence, the collision rate increases as the root-mean- square projection of the chain dimension in the deformation direction, ${ }^{36} \sqrt{l_{\mathrm{c}}{ }^{2}+\tau_{\mathrm{s}}\left(\kappa+\kappa^{\mathrm{T}}\right):\langle\underline{Q Q\rangle}}$ increases. Specifically, the

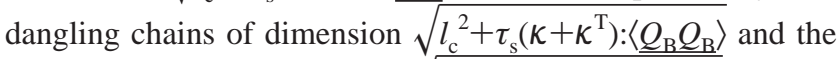
bridging chains of dimension $\sqrt{l_{\mathrm{c}}^{2}+\tau_{\mathrm{s}}\left(\kappa+\kappa^{\mathrm{T}}\right):\left\langle\underline{\left.Q_{A} Q_{A}\right\rangle}\right.}$ are created. Here, $\tau_{\mathrm{s}}$ is the characteristic interaction time of hydrophobic ends with the surrounding fluid medium comprising a lattice of attractive micelles. The probability rate of creation of active chains can then be determined as

$$
\begin{aligned}
L(Q, t) \approx \frac{1}{\tau_{\mathrm{E}}} \frac{1-p_{\mathrm{eq}}}{l_{\mathrm{c}}} \sqrt{\left.l_{\mathrm{c}}^{2}+\tau_{\mathrm{s}}\left(\kappa+\kappa^{\mathrm{T}}\right): \underline{\left\langle Q_{\mathrm{B}} Q_{\mathrm{B}}\right.}\right\rangle}+ \\
\frac{1}{\tau_{\mathrm{E}} \frac{p_{\mathrm{eq}}}{l_{\mathrm{c}}} \sqrt{l_{\mathrm{c}}^{2}+\tau_{\mathrm{s}}\left(\kappa+\kappa^{\mathrm{T}}\right):\left\langle\underline{Q_{\mathrm{A}} Q_{\mathrm{A}}}\right\rangle}}
\end{aligned}
$$

where $p_{\text {eq }}=\int_{\langle a|}^{\infty} 4 \pi r^{2} \exp \left(-0.5 r^{2}\right) \mathrm{d} r / \int_{0}^{\infty} 4 \pi r^{2} \exp \left(-0.5 r^{2}\right) \mathrm{d} r$ denotes the equilibrium probability of chains lying at $|r|>\langle a\rangle$ (the average distance between micelles). The first term accounts for the ejected loops, and the second term accounts for the temporally ejected bridging chains. Equation 20 is nondimensionalized to yield

$$
\begin{array}{r}
\bar{L}(Q, t)=\bar{h}\left(c, M_{\mathrm{w}}\right)\left\{\left(1-p_{\mathrm{eq}}\right) \sqrt{1+G_{\mathrm{m}}\left(\kappa+\kappa^{\mathrm{T}}\right): \mathbf{B}}+\right. \\
\left.p_{\mathrm{eq}} \sqrt{1+G_{\mathrm{m}}\left(\kappa+\kappa^{\mathrm{T}}\right): \mathbf{A}}\right\}
\end{array}
$$

where $G_{\mathrm{m}}=\tau_{\mathrm{s}} / \tau_{\mathrm{E}}$ is a dimensionless constant and $\bar{h}\left(c, M_{\mathrm{w}}\right)$ is a monotonic function of polymer concentration and molecular weight. The form of $\bar{h}\left(c, M_{\mathrm{w}}\right)$ is presented in the next section. The parameter $G_{\mathrm{m}}$ represents the ratio of the interaction time of the hydrophobic end to the characteristic thermal time scale for ejection of hydrophobes. This single parameter characterizing the dependence of the creation process on the nonlinear deformation rate will depend on the specific characteristics of the fluid (e.g., molecular composition and concentration). This completes the set of equations specifying the constitutive response of a model telechelic polymer. For completeness, we summarize the model in Table 2.

We now explore some consequences of the set of equations derived in this section by computing the stress response in steady shear and small-amplitude oscillatory shear flows.

\section{Model Results}

3.1. Equilibrium Composition and Conformation Tensor. In the absence of flow, the number of bridging and looped chains is in dynamical equilibrium. By setting $\kappa=0$, we solve eqs $12-15$ for the equilibrium fraction of bridging chains $\phi_{\mathrm{eq}}$ and for the equilibrium conformation tensors $\mathbf{A}_{\mathrm{eq}}$ and $\mathbf{B}_{\mathrm{eq}}$ for the bridges and danglers to obtain

$$
\frac{v_{\mathrm{A}}}{v}=\phi_{\mathrm{eq}}=\frac{\bar{L}\left(Q_{\mathrm{eq}}{ }^{2}\right)}{\bar{L}\left(Q_{\mathrm{eq}}{ }^{2}\right)+\bar{M}\left(Q_{\mathrm{eq}}{ }^{2}\right)}
$$

and

$$
\mathbf{A}_{\mathrm{eq}}=\boldsymbol{\delta} \text { and } \quad \mathbf{B}_{\mathrm{eq}}=\boldsymbol{\delta}
$$

This results in an isotropic stress tensor $\boldsymbol{\sigma}_{\text {eq }}=\boldsymbol{\delta}$. Equation 22 shows that the equilibrium fraction of bridging chains is the ratio of the probability rate of its formation to the sum of the creation probability rates of bridging and dangling chains. This is consistent with the partition function approach of statistical thermodynamics and the Monte Carlo simulations of Annable 
Table 2. Transient Network Constitutive Model for Telechelic Polymers $^{a}$

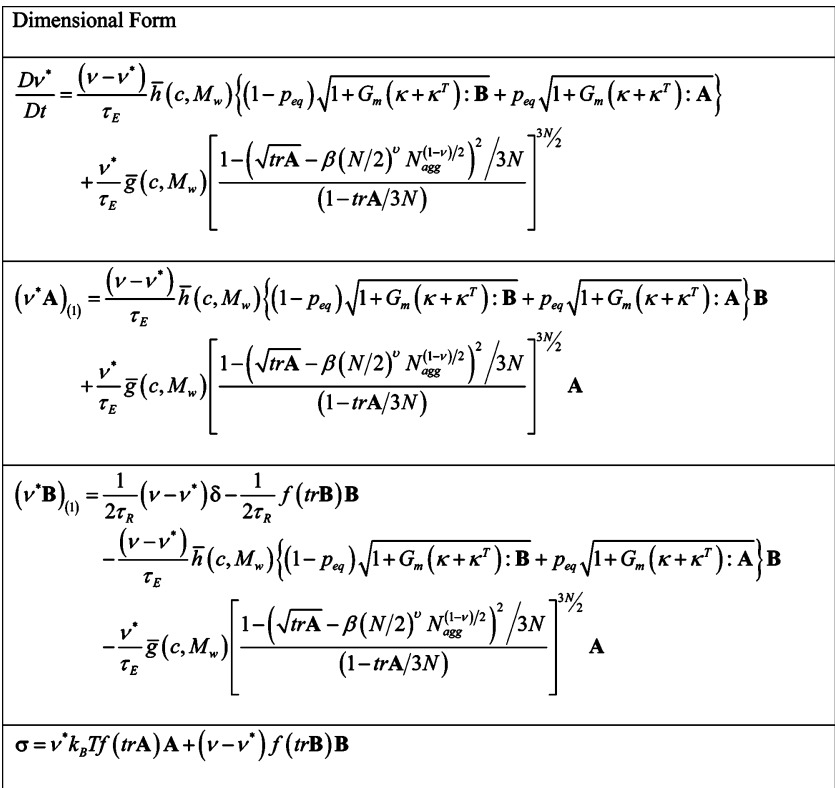

${ }^{a} f(\operatorname{tr} \mathbf{A})=1 /(1-\operatorname{tr} \mathbf{A} / 3 N), f(\operatorname{tr} \mathbf{B})=1 /(1-\operatorname{tr} \mathbf{B} / 3 N)$, and $\bar{g}\left(c, M_{\mathrm{w}}\right)$ and $\bar{h}\left(c, M_{\mathrm{w}}\right)$ are numerical constants that scale with molecular weight; $G_{\mathrm{m}}$ is a dimensionless constant.

and co-workers. ${ }^{5}$ Note that the conformation tensors are independent of the fraction of bridging chains due to the normalization procedure.

3.2. Zero-Shear Viscosity and First Normal Stress Coefficient. We now consider weak flows under steady-state conditions. We explore this by linearizing $\mathbf{A}, \mathbf{B}$, and $\phi$ to a firstorder perturbation in the shear rate $\dot{\gamma}$ and then solving the governing eqs $12-16$. We then substitute the leading order terms in the equation for the stress tensor (eq 10) to obtain the following expression for the dimensionless zero-shear-rate viscosity:

$$
\frac{\eta_{0}}{v k_{\mathrm{B}} T \tau_{\mathrm{E}}}=\frac{\phi_{\mathrm{eq}}}{\bar{M}\left(Q_{\mathrm{eq}}{ }^{2}\right)}+\frac{1}{2} \frac{\tau_{\mathrm{R}}}{\tau_{\mathrm{E}}} \frac{1}{1-\phi_{\mathrm{eq}}}
$$

The first term is the stress contribution arising from the bridging chains, and the second term comes from the dangling chains. This clearly shows that if the Rouse relaxation time of the temporary dangling chain is very short compared to the time scale associated with ejection of hydrophobic ends, then the dangling chains will have negligible contribution to the shear stress. This limit was considered by Tanaka and Edwards. ${ }^{28-31}$ We shall discuss the dependence of the zero-shear-rate viscosity on the polymer concentration and molecular weight later in this section.

By collecting the terms at second order in the perturbation expansion, we can evaluate the first normal stress coefficient as

$$
\begin{aligned}
\frac{\Psi_{10}}{v k_{\mathrm{B}} T \tau_{\mathrm{E}}{ }^{2}}=\frac{2 \phi_{\mathrm{eq}}}{\bar{M}^{2}\left(Q_{\mathrm{eq}}{ }^{2}\right)}+2\left(\frac{\tau_{\mathrm{R}}}{\tau_{\mathrm{E}}}\right) \frac{\phi_{\mathrm{eq}}}{1-\phi_{\mathrm{eq}}} \frac{1}{\bar{M}\left(Q_{\mathrm{eq}}{ }^{2}\right)}+ \\
\frac{1}{2}\left(\frac{\tau_{\mathrm{R}}}{\tau_{\mathrm{E}}}\right)^{2} \frac{1}{\left(1-\phi_{\mathrm{eq}}\right)^{2}}
\end{aligned}
$$

where $\phi_{\text {eq }}$ is given by eq 22 . Note that the second normal stress coefficient $\Psi_{20}=0$ as expected from this preaveraged closed system of equations.
3.3. Small-Amplitude Oscillatory Shear Response. Next, we probe the linear viscoelastic response to a small amplitude oscillatory shear deformation which may be represented in complex form $\gamma=\gamma^{0} \mathrm{e}^{i \omega t}$. Here, $\gamma^{0}$ is the (real, positive) amplitude of the oscillatory shear strain and $\omega$ is the dimensional circular frequency. For small deformations, the conformation tensors $\mathbf{A}$ and $\mathbf{B}$ and the dimensionless shear stress $T_{12}$ are assumed to oscillate with the same frequency, but not necessarily in phase with the shear strain: $\mathbf{A}=\mathbf{A}_{\mathrm{eq}}+\mathbf{A}^{\prime} \mathrm{e}^{i \omega t}, \mathbf{B}=\mathbf{B}_{\mathrm{eq}}+$ $\mathbf{B}^{\prime} \mathrm{e}^{i \omega t}$, and $T_{12}=T_{12}^{\prime} \mathrm{e}^{i \omega t}$ where $\mathbf{A}^{\prime}, \mathbf{B}^{\prime}$, and $T^{\prime}{ }_{12}$ are the leading order responses and can be complex. Substituting these perturbations into eqs $12-16$, we obtain the following expression for the dimensionless complex viscosity $\eta^{*}\left(=\eta^{\prime}-i \eta^{\prime \prime}\right)$

$$
\begin{aligned}
& \frac{\eta^{*}}{v k_{\mathrm{B}} T \tau_{\mathrm{E}}}= \\
& \frac{2 \phi_{\mathrm{eq}}+\left(\frac{\tau_{\mathrm{R}}}{\tau_{\mathrm{E}}}\right)\left\{\bar{L}\left(Q_{\mathrm{eq}}{ }^{2}\right)+\bar{M}\left(Q_{\mathrm{eq}}{ }^{2}\right)+i \omega \tau_{\mathrm{E}}\right\}}{2\left\{i \omega \tau_{\mathrm{E}}+\bar{M}\left(Q_{\mathrm{eq}}{ }^{2}\right)\right\}+\left(\frac{\tau_{\mathrm{R}}}{\tau_{\mathrm{E}}}\right)\left\{i \omega \tau_{\mathrm{E}}\left[\bar{L}\left(Q_{\mathrm{eq}}{ }^{2}\right)+\bar{M}\left(Q_{\mathrm{eq}}{ }^{2}\right)\right]-\left(\omega \tau_{\mathrm{E}}\right)^{2}\right\}}
\end{aligned}
$$

By gathering real and imaginary parts, eq 26 results in the following closed-form expressions for the dynamic moduli $G^{\prime}$ and $G^{\prime \prime}$ :

$$
\frac{G^{\prime}(\omega)}{v k_{\mathrm{B}} T}=\frac{R Q-S P}{P^{2}+Q^{2}} \quad \text { and } \quad \frac{G^{\prime \prime}(\omega)}{v k_{\mathrm{B}} T}=\frac{R P+Q S}{P^{2}+Q^{2}}
$$

where $P=-\left(\omega \tau_{\mathrm{E}}\right)^{2} \tau_{\mathrm{R}} / \tau_{\mathrm{E}}+2 \bar{M}\left(Q_{\mathrm{eq}}{ }^{2}\right), Q=2 \omega \tau_{\mathrm{E}}+\omega\left[\bar{L}\left(Q_{\mathrm{eq}}{ }^{2}\right)\right.$ $\left.+\bar{M}\left(Q_{\mathrm{eq}}{ }^{2}\right)\right] \tau_{\mathrm{R}}, R=2 \phi_{\mathrm{eq}} \omega \tau_{\mathrm{E}}+\left\{\bar{L}\left(Q_{\mathrm{eq}}{ }^{2}\right)+\bar{M}\left(Q_{\mathrm{eq}}{ }^{2}\right)\right\} \tau_{\mathrm{R}} \omega$, and $S=\tau_{\mathrm{R}}\left(\omega \tau_{\mathrm{E}}\right)^{2} / \tau_{\mathrm{E}}$. The nonuniform dependence of the probability rate of dissociation and association on the chain end-to-end distance crucially affects the dynamic moduli, leading to deviation from the conventional form for parallel superposition of linear Maxwell elements. Nevertheless, eq 27 shows that more than one time scale can be important in the linear viscoelastic deformation of telechelic polymers.

3.4. Analytical Solutions in the Limit $\boldsymbol{\tau}_{\mathrm{R}} / \boldsymbol{\tau}_{\mathrm{E}} \rightarrow \boldsymbol{0}$. We now explore some consequences of the set of equations derived above by assuming fast relaxation of the dangling chains. If the relaxation is faster than the hydrophobe dissociation rate $\tau_{\mathrm{R}} \ll$ $\tau_{\mathrm{E}}$, then the average orientation of dangling chains at any time will always be near the equilibrium value. This results in uncoupling of the nonlinear equations for the bridging and dangling chains. Hence, the material functions can be written in simpler form as

$$
\begin{gathered}
\frac{\eta_{0}}{v k_{\mathrm{B}} T \tau_{\mathrm{E}}}=\frac{\phi_{\mathrm{eq}}}{\bar{M}\left(Q_{\mathrm{eq}}{ }^{2}\right)} \\
\frac{\Psi_{10}}{v k_{\mathrm{B}} T \tau_{\mathrm{E}}{ }^{2}}=\frac{2 \phi_{\mathrm{eq}}}{\bar{M}^{2}\left(Q_{\mathrm{eq}}{ }^{2}\right)} \\
\frac{G^{\prime}(\omega)}{v k_{\mathrm{B}} T}=\phi_{\mathrm{eq}} \frac{\left(\omega \tau_{\mathrm{E}}\right)^{2}}{\bar{M}^{2}\left(Q_{\mathrm{eq}}{ }^{2}\right)+\left(\omega \tau_{\mathrm{E}}\right)^{2}} \quad \text { and } \\
\frac{G^{\prime \prime}(\omega)}{v k_{\mathrm{B}} T}=\phi_{\mathrm{eq}} \frac{\bar{M}\left(Q_{\mathrm{eq}}{ }^{2}\right) \omega \tau_{\mathrm{E}}}{\bar{M}^{2}\left(Q_{\mathrm{eq}}{ }^{2}\right)+\left(\omega \tau_{\mathrm{E}}\right)^{2}}
\end{gathered}
$$

Hence, in small-amplitude oscillatory shear, our model reduces to a linear Maxwell model with an effective relaxation time 


$$
\tau_{\mathrm{eff}}=\tau_{\mathrm{E}} / \bar{M}\left(Q_{\mathrm{eq}}{ }^{2}\right)
$$

that depends on the dimensionless destruction rate, which itself depends on polymer concentration and molecular weight. We can easily verify that the zero-frequency viscosity $\lim _{\omega \rightarrow 0}\left(G^{\prime \prime} / \omega\right)=\eta_{0}$ is the same as the zero-shear rate viscosity, as expected. Furthermore, the storage modulus, in the limit $\lim _{\omega \rightarrow 0}\left\{G^{\prime} / \omega^{2}\right\}=\Psi_{10} / 2$ gives the result expected from simple fluid theory. We also obtain the Plateau modulus $G_{N}^{0}$ in the limit of high-frequency modulus as $\lim _{\tau_{\text {eff }} \omega \gg 1} G^{\prime}(\omega)=\phi_{\text {eq }}$, which also scales with polymer concentration and molecular weight.

In the limit $\tau_{\mathrm{R}} / \tau_{\mathrm{E}} \rightarrow 0$, we can also solve explicitly for the steady shear viscosity and the steady extensional viscosity as a function of shear rate. The solution is obtained analytically as a function of the trace of the conformation tensor for bridging chains $Q_{\mathrm{A}}^{2}=\operatorname{tr} \mathbf{A}$. The expressions for the steady shear viscosity and steady extensional viscosity are given by

$$
\frac{\eta(W i)}{v k_{\mathrm{B}} T \tau_{\mathrm{E}}}=\frac{\phi}{\bar{M}(\operatorname{tr} \mathbf{A})} f(\operatorname{tr} \mathbf{A})
$$

and

$$
\frac{\eta_{\mathrm{E}}(D e)}{v k_{\mathrm{B}} T \tau_{\mathrm{E}}}=\frac{3 \phi \bar{M}(\operatorname{tr} \mathbf{A})}{(\bar{M}(\operatorname{tr} \mathbf{A})-2 D e)(\bar{M}(\operatorname{tr} \mathbf{A})+D e)} f(\operatorname{tr} \mathbf{A})
$$

where $f(\operatorname{tr} \mathbf{A})=1 /(1-\operatorname{tr} \mathbf{A} / 3 N), \phi=\bar{L}(\operatorname{tr} \mathbf{A}) /(\bar{L}(\operatorname{tr} \mathbf{A})+\bar{M}(\operatorname{tr}$ $\mathbf{A}))$, the Weissenberg number in steady shear is computed as $W i=\dot{\gamma} \tau_{\mathrm{E}}=\bar{M}(\operatorname{tr} \mathbf{A}) \sqrt{(\operatorname{tr} \mathbf{A}-3) / 2}$ and the Deborah number is evaluated as $D e=\dot{\epsilon} \tau_{\mathrm{E}}=\bar{M}(\operatorname{tr} \mathbf{A})(\sqrt{(\operatorname{tr} \mathbf{A}-3)(9 \operatorname{tr} \mathbf{A}-3)}-(\operatorname{tr} \mathbf{A}$ $-3)) /(4 \operatorname{tr} \mathbf{A})$.

3.5. Concentration and Molecular Weight Scaling of $\bar{g}\left(\boldsymbol{c}, \boldsymbol{M}_{\mathrm{w}}\right)$ and $\overline{\boldsymbol{h}}\left(\boldsymbol{c}, \boldsymbol{M}_{\mathrm{w}}\right)$. We construct the molecular weight and concentration scaling for the observed rheological material properties following the arguments of Annable et al. ${ }^{5}$ and Marrucci et al. ${ }^{36}$ The experimental results of Annable and coworkers show that the concentration-dependent relaxation time scales as a function of $c \sqrt{M_{\mathrm{w}}}$, and the theoretical predictions of Marrucci and co-workers suggest that the effective relaxation time scale $\tau_{\text {eff }} \sim\left(c \sqrt{M_{\mathrm{w}}}\right)^{2 / 3}$. Since the effective relaxation time is inversely proportional to the probability rate of dissociation, we obtain from eqs 19 and 31 that

$$
\bar{g}\left(c, M_{\mathrm{w}}\right)=\chi\langle a\rangle^{2}\left[\frac{1-\frac{1}{3 N}}{1-\frac{\left(\sqrt{3}-r_{\mathrm{c}}\right)^{2}}{3 N}}\right]^{3 N / 2}
$$

where $\langle a\rangle$, given by eq 3 , is the average spatial distance between the micelles and $\chi$ is a numerical constant of order unity. The theoretical predictions of Marrucci et al. ${ }^{36}$ also show the augmented dependence of zero shear viscosity on concentration $\left(\eta_{0} \sim c^{5 / 3}\right)$. Following his scaling arguments and using eqs 28 and 34, we approximated the form of $\bar{h}\left(c, M_{\mathrm{w}}\right)$ as

$$
\bar{h}\left(c, M_{\mathrm{w}}\right)=\frac{\xi F\left(M_{\mathrm{w}}\right)}{\langle a\rangle^{3 / 2}}
$$

where $\xi$ is a numerical constant of order unity and $F\left(M_{\mathrm{w}}\right)$ is a function of molecular weight of the polymer which describes the degree of compression of micellar systems as discussed by Semenov et al. ${ }^{10}$ We approximate a simple form for this function to be

$$
F\left(M_{\mathrm{w}}\right)=\frac{M_{\mathrm{w}}^{*}}{M_{\mathrm{w}}}-1
$$

where $M_{\mathrm{w}}^{*}$ is the critical molecular weight of the polymer when the micellar system turns into a completely compressed gel. At this point no further creation of bridging chains is possible.

3.6. Nonlinear Steady Shear Rheology (Model) Predictions. We now examine the predictions of our model (eqs 1216) for steady-state shear flows. Although it is possible to obtain simplified analytical solutions for the material functions for the limit $\tau_{\mathrm{R}} / \tau_{\mathrm{E}} \rightarrow 0$, this is not true in the more general case because of the terms in eqs $12-16$ that are nonlinear in the conformation tensor. We solve these equations numerically as a set of coupled (scalar) ordinary differential equations. That is, we specify the components of the deformation tensor $\kappa+\kappa^{\mathrm{T}}$ and solve eqs 12-16 with a Runge-Kutta routine. Steady-state material functions are obtained as the long time asymptotes of the corresponding material functions following the instantaneous inception of the flow. We neglect the solvent contribution of stress (such a contribution is not significant in a semidilute aqueous solution).

The description of the evolution of material properties depends on various molecular features such as molecular weight $M_{\mathrm{w}}$, aggregation number $N_{\mathrm{agg}}$, number of hydrophobic carbon moieties $n_{\mathrm{c}}$, the concentration of telechelic chains $c$, and the solvent quality. In addition, material properties are functions of the rate of deformation and the temperature of the system. Although the quantitative dependence of the material properties on each of these molecular features can be studied, we specifically focus our attention on the variation with concentration and the number of hydrophobic carbon links. Hence, we examine the predictions of our model for a particular set of molecular properties $N_{\mathrm{agg}}=20, M_{\mathrm{w}}=65300 \mathrm{~g} / \mathrm{mol}$, $T=25^{\circ} \mathrm{C}, c$ ranging from 10 to $40 \mathrm{~kg} / \mathrm{m}^{3}(1-4 \mathrm{wt} \%)$, and $n_{\mathrm{c}}$ ranging from 14 to 16 . In addition, we also fix the numerical values of various model constants as $\beta=0.07, \chi=0.3, \xi=$ 1.2 , and $M_{\mathrm{w}}^{*}=109 \times 10^{3} \mathrm{~g} / \mathrm{mol}$.

An interesting feature of the model is the importance of the deformation-induced creation rate as compared to thermal diffusion rate, given by the parameter $G_{\mathrm{m}}$. Figure 3 a shows the steady-state fraction of bridging chains $\phi$ as a function of the dimensionless shear rate $\dot{\gamma} \tau_{\mathrm{E}}$ for several values of the dimensionless number $G_{\mathrm{m}}$. Here, the polymer concentration and the number of hydrophobic units are $c=2 \mathrm{wt} \%$ and $n_{\mathrm{c}}=16$, respectively. At low shear rates, thermally induced fluctuations predominantly initiate both the destruction and creation of the bridging chains. Since rates for these two processes are initially equal $\left(1 / \tau_{\mathrm{E}}\right)$, the fraction of bridging chains stays constant. With increasing shear rate, both the rate of creation and the rate of stretch-induced destruction of bridging chains increase nonlinearly. The balance between these rates characterizes the shear thickening and shear thinning behavior of the telechelic polymers. When the exit rate of hydrophobic end groups exceeds the collision rate of the dangling end groups with the surrounding micelles, the fraction of bridging chains decreases with increasing shear rate. Hence, for $G_{\mathrm{m}}<1$, the increase in shear rate leads to an increase in the stretching force in the bridging chains, which decouples them from the micellar junction. However, for some telechelic polymeric systems, the rate of deformation-induced association of the dangling end groups increases for a range of shear rates. Hence, for $G_{\mathrm{m}}>1$, the number of elastically active bridges first increases and subsequently decreases with the applied shear rate. 

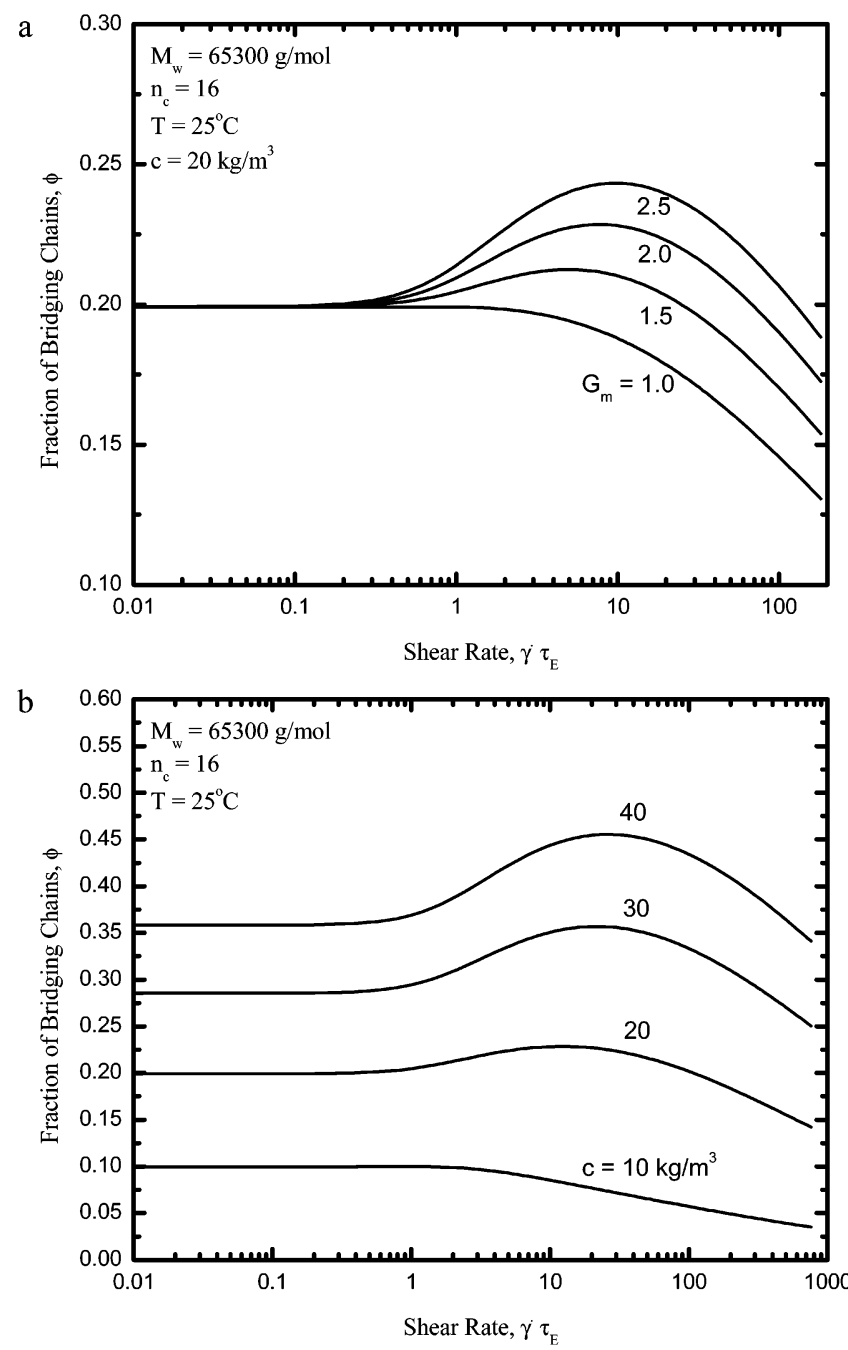

Figure 3. (a) Steady-state fraction of bridging chains $\phi$ as a function of dimensionless shear rates $\dot{\gamma} \tau_{\mathrm{E}}$ for several values of the dimensionless number $G_{\mathrm{m}}$, at a concentration $c=2 \%$. (b) Steady-state fraction of bridges as a function of applied shear rate for polymer concentrations ranging $1-4 \mathrm{wt} \%$.

Figure $3 b$ shows the steady-state fraction of bridges as a function of applied shear rate for concentrations ranging from 1 to $4 \mathrm{wt} \%$. In general, the number of bridging chains stays constant at low values of shear rate, increases at moderate shear rates, and eventually decreases at large values of the shear rate. At low concentrations, only a few bridging and dangling chains is present in the network system. Hence, the molecular associations hardly increase the number of bridging chains in a shearing telechelic polymer system. As the concentration increases, the probability of association of a dangling chain increases, and the maximum value attained by number of bridging chains also increases.

In Figure 4 we present the dimensionless steady shear viscosity as a function of the dimensionless shear rate for different values of polymer concentration $c$. The steady shear viscosity profile captures typical characteristics of telechelic polymers such as a shear-rate-independent viscosity at low shear rates, shear thickening at intermediate shear rates, and severe shear thinning at elevated shear rates. Although the zero-shear viscosity is parameter independent, the amount of shear thickening can be adjusted by tuning the parameter $G_{\mathrm{m}}$. As the concentration increases, the onset of shear thickening and shear thinning shift to lower shear rates. This is consistent with the recent experimental findings of $\mathrm{Ma}$ and Cooper. ${ }^{21}$ The model

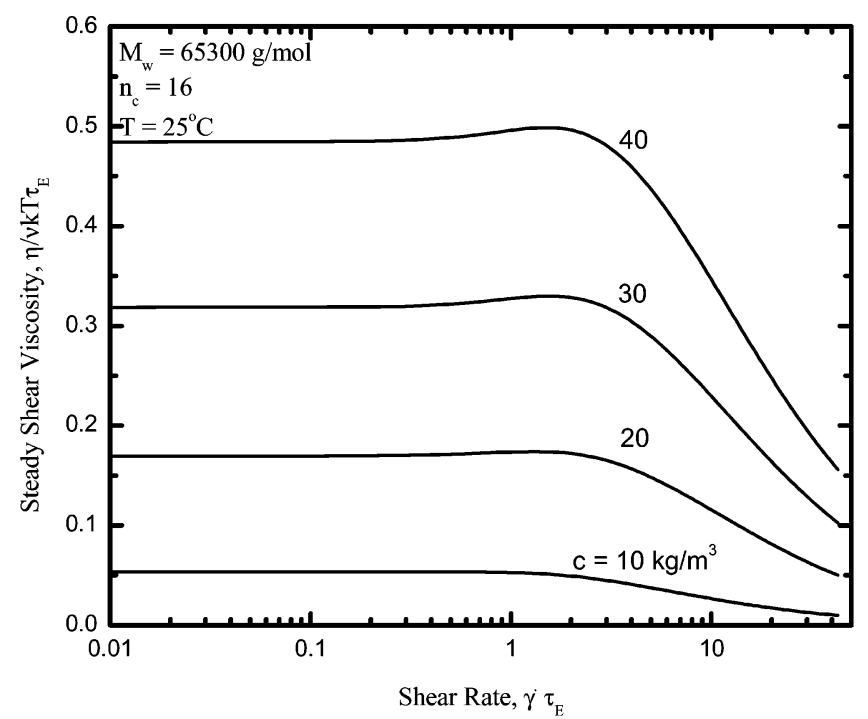

Figure 4. Steady-state shear viscosity as a function of dimensionless shear rates $\dot{\gamma} \tau_{\mathrm{E}}$ for concentrations ranging $1-4 \%$.

predicts corresponding features of the steady shear stress profile such as a constant rate of increase of shear stress at low values of shear rates and a slow rise at high shear rates. For the range of concentrations and molecular weights in the present study, most of the shear thinning that is observed is a result of progressive network deformation rather than network collapse. Analysis of the first normal stress difference profiles further supports these conclusions. The normal stress difference is found to slowly increase in the shear thinning region, and its value is larger than the corresponding shear stress $\left(\tau_{11}-\tau_{22}\right) \geq \tau_{12}$. Hence, the network becomes increasingly elastic at high values of the Weissenberg number. These observations are consistent with recent experimental findings of Sadeghy and James. ${ }^{26}$

3.7. Nonlinear Extensional Rheology (Model) Predictions. We now examine the predictions of our model (eqs 12-16) for uniaxial extensional flows. We solve these equations numerically as a set of coupled (scalar) ordinary differential equations. Figure 5a shows the transient extensional viscosity of a $4 \%$ HEUR solution for various Deborah numbers. After a rapid increase at short times, the extensional viscosity rapidly attains a steady-state value for all values of Deborah number. As the Deborah number increases, the critical time to reach steady state decreases. For comparison, the linear viscoelastic (LVE) extensional viscosity $\eta_{\mathrm{E}}{ }^{+}=3 \eta_{0}\left(1-\exp \left(-t / \tau_{\mathrm{E}}\right)\right)$ is also plotted in Figure 5a. The model clearly predicts a strain hardening behavior for associative polymers. Figure $5 \mathrm{~b}$ shows the steady extensional viscosity as a function of Deborah number for three different sets of $N$ and $n_{\mathrm{c}}$ values that parametrize the PEO chain length and hydrophobe length. The steady extensional profile captures important characteristics of telechelic polymers such as strain-rate-independent viscosity at low extensional rates, strain hardening at intermediate strain rates, and an extensional viscosity decrease at elevated $D e$. The increase in viscosity is due to a strain-induced increase in bridging interactions. The experimental work of Sadeghy and James $^{26}$ also show strain hardening behavior for $0.2<D e<$ 0.8 . For consistency with our initial theoretical model considerations, we have defined the Deborah number as $D e=\tau_{\mathrm{E}} \dot{\epsilon}$; however, we recognize that a more realistic definition of Deborah number should be $D e=\tau_{\text {eff } \dot{\epsilon}}$. This can be easily evaluated using eq 31. As expected, on increasing the hydrophobic strength (stronger micelles) the extensional viscosity rapidly rises. Similarly, a decrease in the length of the bridging 

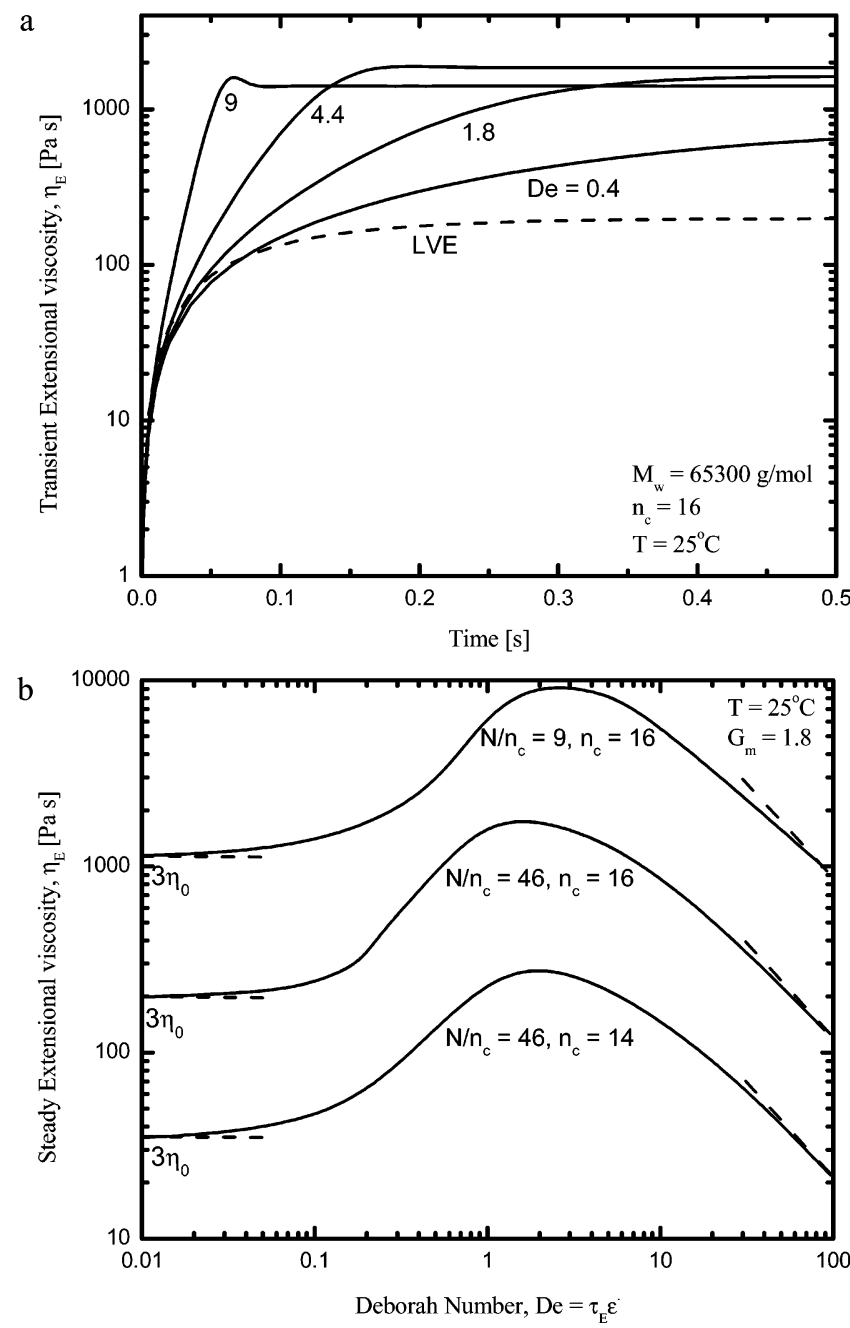

Figure 5. (a) Transient extensional viscosity for $4 \mathrm{wt} \%$ HEUR: model predictions. (b) Steady extensional viscosity as a function of Deborah number for 4 wt \% HEUR: model predictions.

chains leads to an increase in the elongational viscosity due to nonlinear elastic stretching of the chains. Figure $5 \mathrm{~b}$ also shows the analytical solutions (for the limit $\tau_{\mathrm{R}} / \tau_{\mathrm{E}} \rightarrow 0$ ) to be in excellent agreement for small $D e$. Furthermore, at large Deborah numbers, the model predicts an inverse dependence on $D e$, showing that the extensional stress difference saturates. Dashed lines with -1 asymptotic slope are also indicated in the figure.

To summarize, the proposed nonlinear network model is able to predict the majority of commonly observed characteristics of telechelic polymers. In the next section, we proceed to describe our experimental work and then compare the experimental data with model predictions.

\section{Experiments}

The model polymers used in this study were $\mathrm{C}_{16} \mathrm{H}_{33}$ and $\mathrm{C}_{20} \mathrm{H}_{41}$ (i.e., $n_{\mathrm{c}}=16,20$ ) end-capped urethane-coupled poly(oxyethylene) of three different molecular weights. The model HEUR polymers were synthesized and characterized by Dr. R. D. Jenkins. The chemical structure is shown in Figure 6, and the composition details are also summarized. In the first column some simple codes used to represent "specific molecular compositions" are assigned. We also present the estimated molecular properties for these model polymers. We ignore any variation in the aggregation number either with the polymer concentration $^{15}$ or under an applied deformation field. ${ }^{55}$ The procedure describing the synthesis of the HEUR polymers is described in detail by Jenkins et al. ${ }^{56}$ The number-averaged molecular weight, $M_{\mathrm{n}}$, and the polydispersity index of the polymer, $M_{\mathrm{w}} / M_{\mathrm{n}}$, were measured in THF using a standard GPC technique (Shimadzu GPC system). The polymer was stored in the refrigerator at $4{ }^{\circ} \mathrm{C}$ to minimize air oxidation and degradation. The samples were made by mixing a known amount of Millipore Milli-Q distilled water and were kept for 2 days prior to testing to allow the solutions to equilibrate. The relevant concentration-molecular weight regimes are discussed in detail in section 4.1 .

The shear flow experiments were performed using a controlledstress rheometer (AR-1000N, TA Instruments). Different geometries (including cone-and-plate, Couette fixtures) were used to probe the rheological response of the test fluids. Both steady shear and small-amplitude oscillatory tests were performed. For oscillatory testing, the applied strain was kept to amplitudes less than $\gamma^{0} \leq 0.2$ in order to ensure that the tests were within the linear viscoelastic region. Experiments were carried out at temperature of $25^{\circ} \mathrm{C}$ and controlled using a Peltier plate.

The extensional flow measurements were performed using a filament stretching rheometer, and the procedure has been described elsewhere. ${ }^{57-59}$ A nearly cylindrical sample of HEUR polymer solution initially fills the gap between two rigid, circular end plates with diameter $D_{0}=3 \mathrm{~mm}$ and initial separation $L_{0}$ $=1.5 \mathrm{~mm}$. To estimate the effect of gravitational sagging of the samples, the ratio of gravitational to surface tension forces, characterized by the dimensionless Bond number $B_{0}=\rho g D_{0}^{2} /$ $4 \Gamma$, was evaluated. Here, the surface tension $\Gamma$ of the HEUR polymer solutions was measured to be $\Gamma=40 \mathrm{dyn} / \mathrm{cm}$. The Bond number for the fluid samples was kept small $\left(B_{0}=0.55\right)$ to minimize the errors due to gravitational sagging. The end plates were then moved apart to a final separation with an exponentially increasing separation profile, $L(t)=L_{0} \exp (\dot{E} t)$, where $\dot{E}$ is the strain rate. While the fluid filament was being stretched the evolution in the tensile force and mid-filament diameter were measured simultaneously. Although the imposed filament length increase is exponential, the measured evolution in the mid-filament diameter was observed to be far from ideal (i.e., it was not described by the homogeneous deformation of a cylindrical element $D_{\text {mid }}(t)=D_{0} \exp (-0.5 \dot{\epsilon} t)$ ) due to additional shearing motion induced at either end plate. Hence, filament stretching experiments are performed twice for each sample, first using an imposed separation profile, $L(t)=L_{0}$ exp$(\dot{E} t)$, to construct a "master curve" 58 and second using a corrected separation profile, $L(t)=L_{0} \exp \left(2 \ln \left(D_{0} / D_{\text {mid }}(t)\right) t\right)$. These so-called "type III" tests ${ }^{58}$ resulted in ideal uniaxial extension of the HEUR samples with a constant deformation rate at the midplane.

4.1. Concentration-Molecular Weight Regime. The interentanglement and recombination of hydrophobic chains under quiescent conditions depends on the molecular weight and volume fraction of the polymer. In dilute solutions the number of bridges is exponentially small; thus, the attraction energy for the recombination of hydrophobic chains is weak. However, the excluded volume repulsion is also small in this region, and the interaction energy is dominated by attraction. ${ }^{8,9}$ For perfectly end-capped systems there is a tendency for phase separation to be observed in this dilute regime. ${ }^{7-9}$ As the concentration of polymer increases, the attraction between the micelles increases; however, the excluded volume repulsion between chains also increases. The coronas of neighboring micelles become compressed. When the concentration is further increased, the attraction energy between two flowers is large for large aggregation number so that the micelles have a tendency to 


Hydrophobe
\begin{tabular}{|l|l|l|l|l|l|l|l|l|}
\hline NAME & Nomenclature & $\mathbf{n}_{\mathbf{c}}$ & $\mathbf{x}$ & $\mathbf{y}$ & $\mathbf{N}$ & $\mathbf{M}_{\mathbf{n}}(\mathbf{1})$ & $\mathbf{M}_{\mathbf{w}} \mathbf{( 2 )}$ & $\mathbf{M}_{\mathbf{w} / \mathbf{M}_{\mathbf{n}}}$ \\
\hline HEUR22-2 & C16PEO34K & 16 & 186 & 4 & 744 & 34,200 & 65,300 & 1.91 \\
\hline HEUR22-4 & C16PEO67K & 16 & 186 & 8 & 1488 & 67,600 & 93,000 & 1.38 \\
\hline HEUR22-6 & C16PEO100K & 16 & 186 & 12 & 2232 & 100,400 & 105,400 & 1.05 \\
\hline HEUR23 & C20PEO51K & 20 & - & - & 1122 & 51,000 & 86,000 & 1.68 \\
\hline
\end{tabular}

Note: (1) Number average molecular weight calculated from reaction stoichiometry

(2) Weight average molecular weight determined from GPC in THF

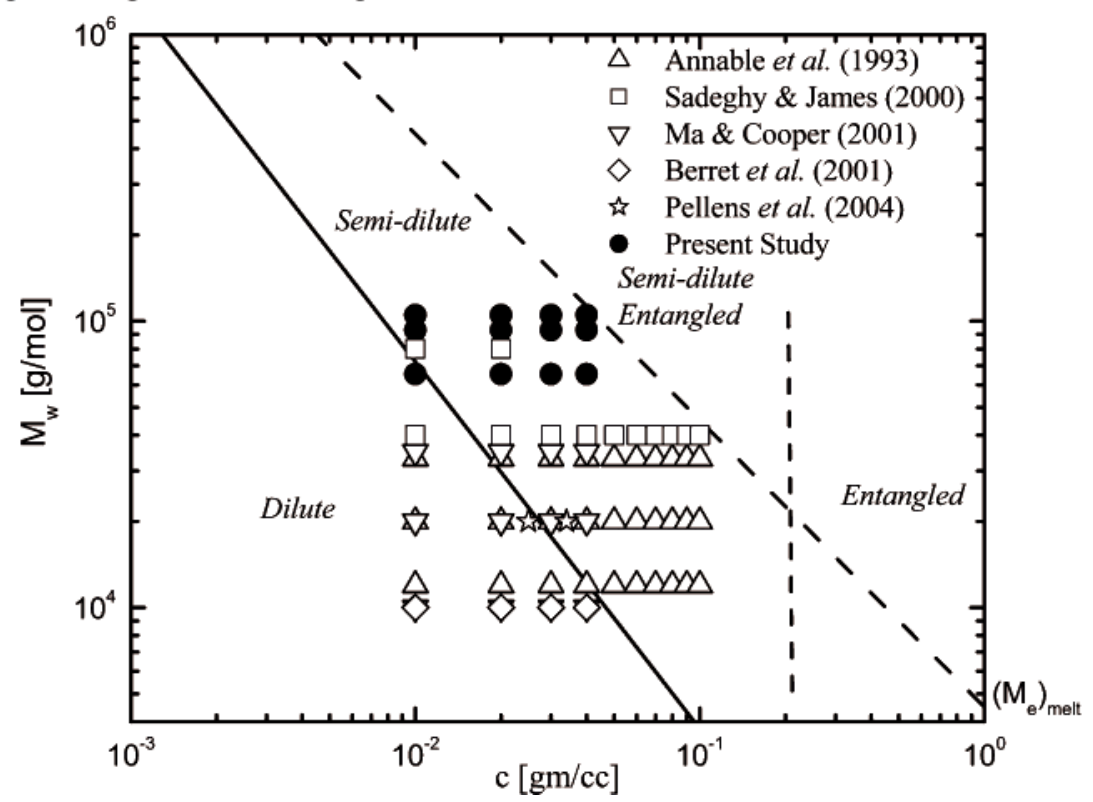

Figure 6. Chemical structure of model HEUR polymers and a plot of concentration vs molecular weight showing various regimes of associative polymer solutions together with the range of solutions studied by other workers.

entangle. Hence, the net interaction energy ${ }^{10}$ between the hydrophobic units and the micelles is expected to vary from dilute to semidilute and from semidilute to entangled polymer solutions. Graessley ${ }^{60}$ represented the viscoelastic behavior of polymer solutions in a succinct manner by constructing a concentration-molecular weight diagram. Following this idea, we compute the overlap concentration $c^{*}$ for the PEO chains, which separates the dilute region from the semidilute region by $c^{*}=0.77 /[\eta]$, where $[\eta]$ is the intrinsic viscosity of the solution. Similarly, the boundary between unentangled and entangled regimes is described by $\left(M_{\mathrm{e}}\right)_{\text {soln }}=\left(M_{\mathrm{e}}\right)_{\text {melt }} / c$ with $\left(M_{\mathrm{e}}\right)_{\mathrm{melt}}=4500 \mathrm{~g} / \mathrm{mol}$ for PEO. ${ }^{2}$ The intrinsic viscosity of the poly(ethylene oxide) in a good solvent such as water can be approximated using $[\eta]=0.0125 M_{\mathrm{w}}{ }^{0.78} \mathrm{~mL} / \mathrm{g} .{ }^{61}$ Figure 6 shows the concentration-molecular weight diagram distinguishing various regimes for PEO-based HEUR solutions. Also, shown are the concentration and molecular weights of model associative polymers used in various other recent studies. Most of the published literature results lie near the dilute/semidilute regime. The viscoelastic properties of semidilute solutions are very sensitive to solvent quality and the precise concentration of dissolved polymer. This is one possible reason for the large variation in properties of HEUR polymers reported in some previous studies.

4.2. Comparison with Shear Rheology Experiments. It is known that the viscosity of hydrophobically end-capped poly(ethylene oxide) solution is much higher than that of an unmodified PEO solution at the same concentration and also depends more strongly on the polymer concentration. This viscosity enhancement is convincing evidence of the association of the hydrophobic end groups in aqueous solution. To evaluate the zero-shear-rate viscosity of the fluid in steady shear flow, we use eq 24, where the equilibrium fraction of bridging chains is given by eq 22 . The equilibrium values of the formation rate $L_{\mathrm{eq}}$ and destruction rate $M_{\mathrm{eq}}$ reduce from eqs 21 and 19, respectively, to

$$
\begin{gathered}
L_{\mathrm{eq}}=\xi\left(\frac{M_{\mathrm{w}}^{*}}{M_{\mathrm{w}}}-1\right) /\left(\frac{3 N_{\mathrm{agg}} M_{\mathrm{w}}}{8 \pi c N_{\mathrm{A}}}\right)^{1 / 2} \\
M_{\mathrm{eq}}=\chi\left(\frac{3 N_{\mathrm{agg}} M_{\mathrm{w}}}{8 \pi c N_{\mathrm{A}}}\right)^{2 / 3}
\end{gathered}
$$

Finally, the constants $\chi$ and $\xi$ are set to the values specified in 


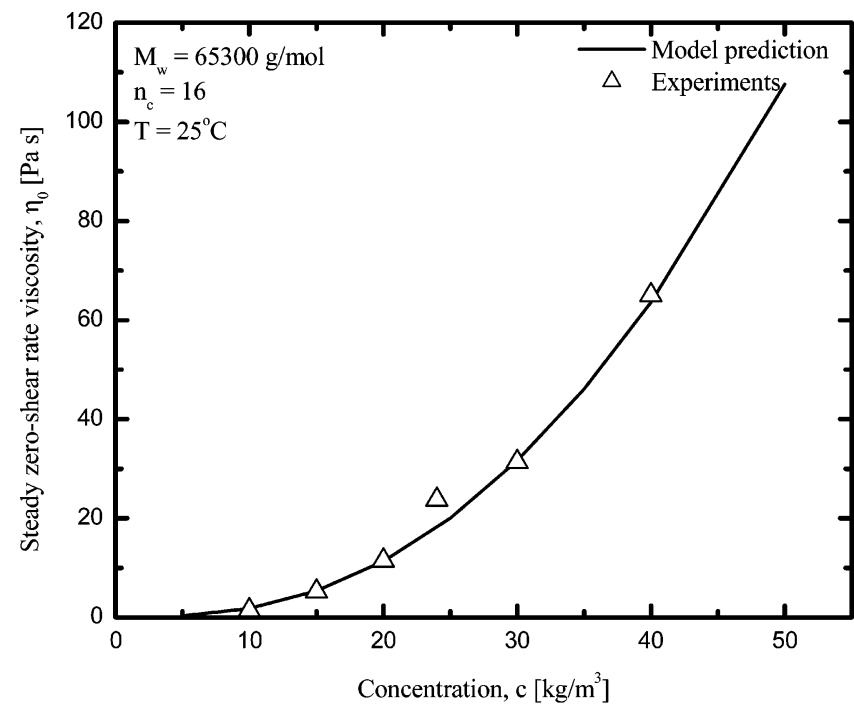

Figure 7. Effect of HEUR concentration on the measured steady zeroshear rate viscosity for HEUR22-2 model polymers.

section 3.6. Figure 7 shows the effect of HEUR concentration on the steady zero-shear rate viscosity for a series of model polymers at $25^{\circ} \mathrm{C}$. Both the experimentally measured data and the model predictions show dramatic increases in the viscosity with increase in concentration. The predicted scaling $\eta_{0} \sim c^{2.75}$ of the model provides a good description of the experiments. It is important to note that the zero-shear viscosity is independent of the nonlinear parameter $G_{\mathrm{m}}$ in the model.

The viscoelastic response of a $2 \mathrm{wt} \%$ HEUR22-2 sample to a small-amplitude oscillatory shear deformation is shown in Figure $8 \mathrm{a}$. The experimental data are fitted using the model functions given by eq 30. Note that once again the linear viscoelastic response is independent of the model parameter $G_{\mathrm{m}}$. As can be seen, the model describes the frequency dependence of both the elastic storage modulus and viscous loss modulus data very well. The viscous and elastic moduli cross at a critical frequency $\omega^{*}$ which is commonly taken to be the effective relaxation time of the active network. Equating the expression of $G^{\prime}$ and $G^{\prime \prime}$ from eq 30 leads to

$$
\omega^{*}=\frac{1}{\tau_{\text {eff }}}=\frac{M_{\mathrm{eq}}}{\tau_{\mathrm{E}}}=\frac{\chi\left(\frac{3 N_{\mathrm{agg}} M_{\mathrm{w}}}{8 \pi c N_{\mathrm{A}}}\right)^{2 / 3}}{\tau_{\mathrm{E}}}
$$

where we have used eq 38 in the final equality. This effective time constant scales as $\tau_{\text {eff }} \sim c^{2 / 3}$. It should be noticed that the Rouse relaxation time $\tau_{\mathrm{R}}=5 \times 10^{-5} \mathrm{~s}$ for HEUR22-2 is many orders of magnitude smaller than the network time $\tau_{\text {eff }}=0.076$ s. Under these circumstances the dangling chains are essentially always in their equilibrium conformation. Thus, only bridging chains (with $\phi_{\mathrm{eq}}=0.2$ ) contribute significantly to the total polymeric stress in the system, and the relaxation process of the temporary dangling chains has a negligible contribution to the viscoelastic response.

Figure $8 \mathrm{~b}$ shows the shear rate dependence of the steadyshear viscosity of the same $2 \mathrm{wt} \%$ HEUR22-2 sample. After showing Newtonian behavior at low shear rates, the data exhibit a moderate shear thickening region and a maximum in viscosity at $\dot{\gamma} \sim 10 \mathrm{~s}^{-1}$ followed by pronounced shear thinning at high shear rates. The model predictions agree remarkably well with the experimental data and capture all of the characteristics of the shear rate dependence. Here, the amount of shear thickening is tuned by adjusting the rate of creation of active junctions through the value of $G_{\mathrm{m}}$ (the single nonlinear model parameter). Hence, an increase in the number of active chains with shear rate leads to the shear thickening of these HEUR solutions. At higher rates the increased rate of creation is overcome by the nonlinear stretching of the elastically active chains which leads to a rapid increase in the junction pull-out rate. Also, shown in the figure is the frequency-dependent dynamic or complex viscosity $\left|\eta^{*}(\omega)\right|$ obtained from small-amplitude oscillatory shear experiments. The steady shear viscosity and dynamic viscosity are equal at low frequencies, whereas at higher frequencies the complex viscosity shows a much stronger dependence on frequency than the steady shear data exhibits with shear rate. This deviation from the Cox-Merz rule has been observed experimentally $y^{3,4}$ and has been noted as one of the primary rheological features of telechelic polymer rheology. In small-amplitude deformations, the contribution of shearenhanced aggregation of micellar junctions given by eq 21 is expected to be negligible.

As the concentration of HEUR chains is increased, we expect the effective time constant to increase $\left(\tau_{\text {eff }} \sim c^{2 / 3}\right)$, the total zeroshear rate viscosity of solution to increase $\left(\eta_{0} \sim c^{2.75}\right)$, and the plateau modulus to increase $\left(G_{N}^{0} \sim \phi_{\text {eq }}\right)$. The number of active bridging chains and the effective time constant increase to $\phi_{\mathrm{eq}}=0.35$ and $\tau_{\text {eff }}=0.12 \mathrm{~s}$, respectively, for $4 \mathrm{wt} \%$ HEUR22-2 solutions. The model captures each of these features as we show in Figures 8(c) and (d) for the 4 wt \% HEUR22-2 solution. In addition to the expected trends noted above, we find that the shear thickening observed at intermediate concentrations is greatly reduced. This effect can be captured by varying the parameter $G_{\mathrm{m}}$, as shown in Figure 3a. The anisotropic creation rate thus appears to be concentration-dependent. We thus fit the data by decreasing the value of the nonlinear model parameter $G_{\mathrm{m}}=1.8$. The model predictions again show a good agreement with the experiments. The disappearance of a shear thickening regime is captured in the model by reducing the value of the nonlinear parameter $G_{\mathrm{m}}$. Hence, at high concentrations when the distance between neighboring micelles is small, there exist a large number of active bridging chains and the flowinduced anisotropy in the number of active bridging chains is minimal.

Having verified the concentration dependence of the viscometric properties both experimentally and numerically, subsequent experiments were performed for HEUR solutions with longer PEO chains $\left(M_{\mathrm{w}}=93000 \mathrm{~g} / \mathrm{mol}\right)$ and identical aliphatic end groups $\left(n_{\mathrm{c}}=16\right)$. Hence, the solutions are of lower relative hydrophobicity and contain weaker micelles leading to lower overall viscosity values. Figure 9 a shows the linear viscoelastic response of a $3.8 \mathrm{wt} \%$ HEUR22-4 sample along with the model prediction. The observed frequency dependence of the loss and elastic moduli is once again well described by the model. Only $14.5 \%$ of chains $\left(\phi_{\text {eq }}=0.145\right)$ carry stress, and the effective time constant for the network is $\tau_{\text {eff }}=0.13 \mathrm{~s}$. Figure $9 \mathrm{~b}$ shows the measured steady shear viscosity for various applied shear rates. The data are in fair agreement with the predictions of model over entire range of shear rate. It is important to emphasize that the evaluation of the zero-shear-rate viscosity requires no free parameter. The scaling is predicted by eq 29 and given fixed values of $M_{\mathrm{w}}$ and $c$. The magnitude of the shear thickening is once again adjusted using the single model parameter $G_{\mathrm{m}}$. Good agreement is also obtained for the $6.8 \mathrm{wt}$ $\%$ HEUR22-6 sample with $\eta_{0}=24.7 \mathrm{~Pa} \cdot \mathrm{s}, \tau_{\text {eff }}=0.19 \mathrm{~s}$, and $G_{\mathrm{m}}=3.1$. However, space precludes us from presenting the results here. 

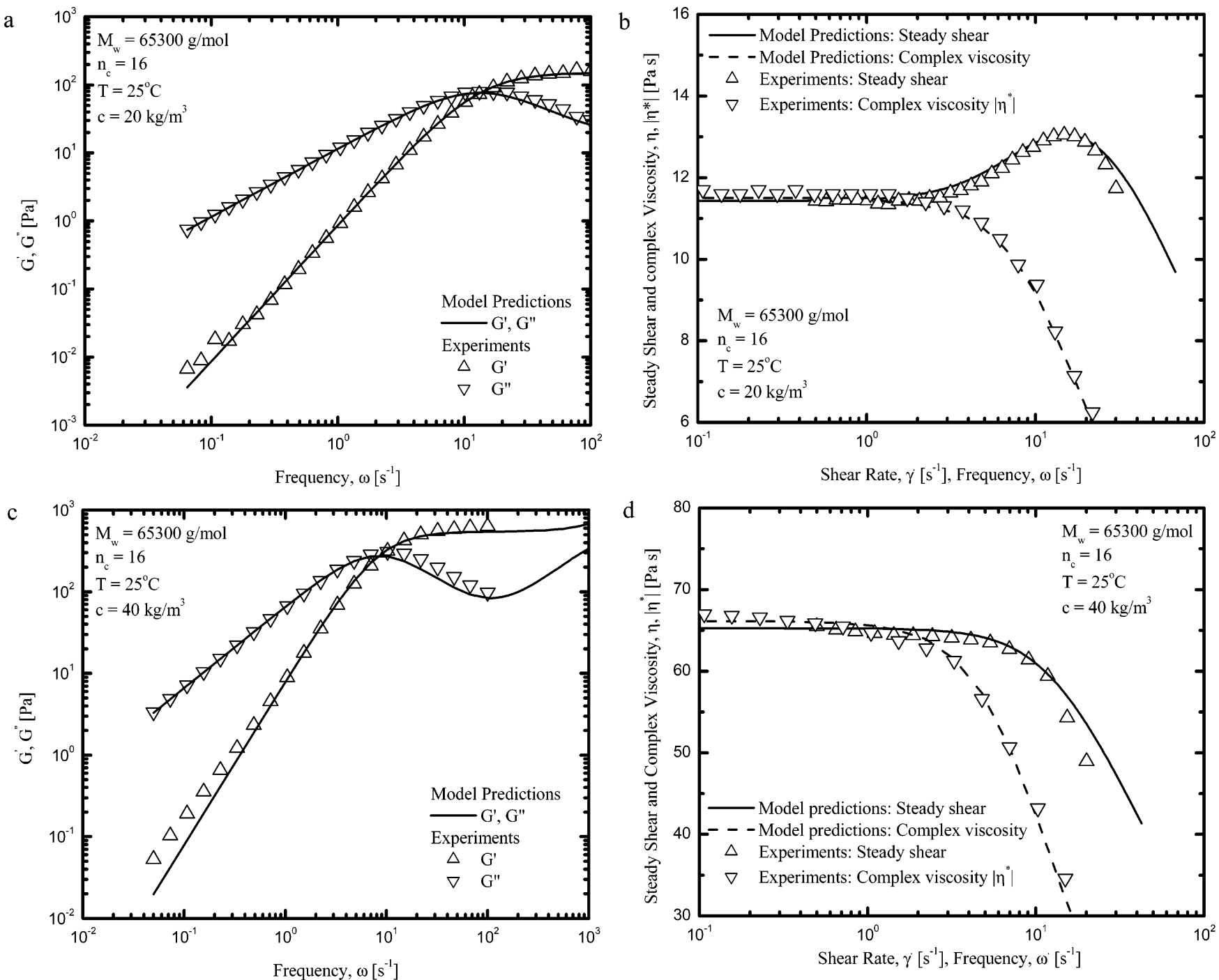

Figure 8. (a) Comparison of the linear viscoelastic response of $2 \mathrm{wt} \%$ HEUR22-2 with the model predictions. $\tau_{\mathrm{R}}=5 \times 10^{-5} \mathrm{~s}$ and $\tau_{\mathrm{E}}=0.089$ s. (b) Steady shear viscosity vs shear rate for $2 \mathrm{wt} \%$ HEUR22-2: experiments and model. $G_{\mathrm{m}}=7.3$. The magnitude of complex viscosity vs frequency is also plotted. (c) Comparison of the linear viscoelastic response of $4 \mathrm{wt} \%$ HEUR22-2 with the model predictions. $\tau_{\mathrm{R}}=0.0002 \mathrm{~s}$ and $\tau_{\mathrm{E}}=0.089 \mathrm{~s}$. (d) Steady shear viscosity vs shear rate for $4 \mathrm{wt} \%$ HEUR22-2: experiments and model. $G_{\mathrm{m}}=1.8$.

Further experiments were also performed using 2 wt $\%$ HEUR23 polymers $\left(M_{\mathrm{w}}=86000 \mathrm{~g} / \mathrm{mol}\right)$ with end-caps of higher hydrophobicity $\left(n_{\mathrm{c}}=20\right.$, i.e., longer end groups). For such materials, the activation energy for disengagement increases, and the solution is likely to exhibit strong networkforming properties. Although it can be naively argued that more hydrophobic end groups likely participate in the formation of micelles, in fact, the ratio of hydrophilic to hydrophobic chain segments has increased to $N / n_{\mathrm{c}}=48.57$ from $N / n_{\mathrm{c}}=46.5$ for 2 wt \% HEUR22-2. Hence, we have kept the aggregation number $N_{\text {agg }}$ constant for evaluation of rheological responses. Figure 10a shows the viscoelastic response of 2 wt \% HEUR23 sample to small-amplitude oscillatory shearing deformation. Because the depth of energy well (described in Figure 1) has increased due to the longer hydrophobes, the relaxation time of the network has increased dramatically to $\tau_{\text {eff }}=4.52 \mathrm{~s}$. As a consequence of this shift in the viscoelastic response of the network a second relaxation process becomes visible at high frequencies. $\mathrm{Ng}$ et al. ${ }^{20}$ denote this "the lifetime of the hydrophobic interactions". Analysis of eqs 27 and 30 shows that this mode is associated with the viscoelastic response of the dangling chains (with characteristic time scale $\tau_{\mathrm{R}}$ ). In fact, there is an upturn in both $G^{\prime}$ and $G^{\prime \prime}$ (shown in Figure 10); however, because the plateau modulus of the first mode is 30 times greater than $G^{\prime \prime}{ }_{\min }$, the additional contribution to the storage modulus is negligibly small within the experimentally accessible frequency window ( $\left.\omega \leq 10^{2} \mathrm{rad} / \mathrm{s}\right)$. It is important to note that only $11 \%$ of chains participate as active bridging chains (i.e., there are more looping chains) which leads to a low value of the plateau modulus. The observed dependency of the loss modulus and storage modulus on dynamic frequency is captured remarkably well by the model. Figure $10 \mathrm{~b}$ shows the steady shear viscosity for $2 \mathrm{wt} \%$ HEUR23 sample. After showing Newtonian behavior at low shear rates, the data exhibit a shear thickening region at intermediate shear rates $\dot{\gamma} \sim 0.2 \mathrm{~s}^{-1}$, which is much lower than observed in HEUR22-2 samples. However, the corresponding Weissenberg number is $W i \sim 1$ and is thus comparable to the value for shear thickening in Figure 8b. The model predictions agree well with this experimental data. At high shear rates, the experimental data show much more rapid shear thinning than that predicted by the model. Since the sample shear thins at a rate faster than $\dot{\gamma}^{-1}$ (see dashed line Figure 10b), this is unlikely to be of rheological origin; possible causes for the rapid shear thinning may be either (a) a shear-induced phase separation or (b) slip between the sample and conical fixture of the rheometer. Shear-induced phase separation has been observed in short chains with long hydrophobic end groups $\left(n_{\mathrm{c}}=\right.$ 18) at stresses as small as $10-15 \mathrm{~Pa}^{62}$ Our measurements are 

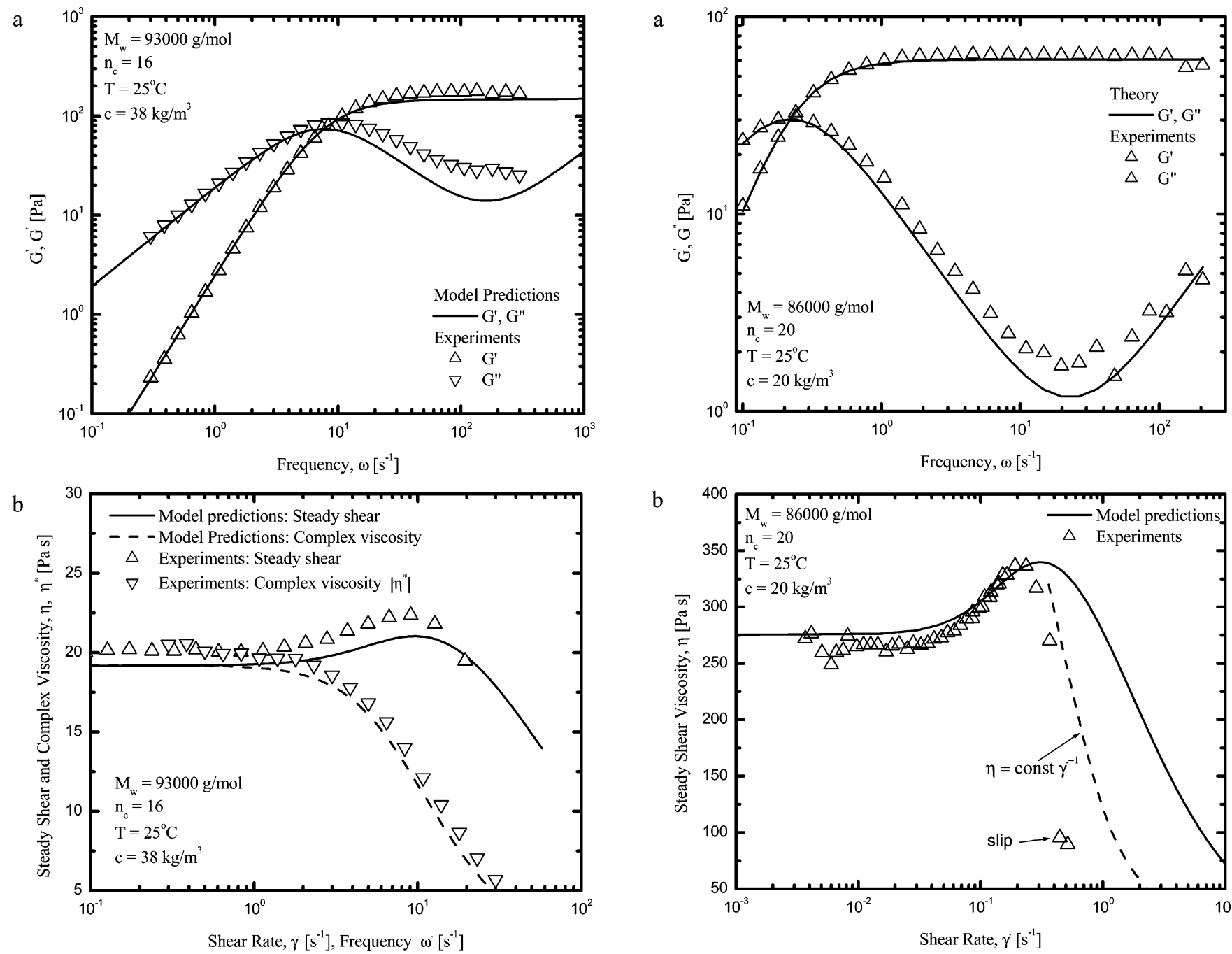

Figure 9. (a) Comparison of the linear viscoelastic response of 3.8 wt $\%$ HEUR22-4 with the model predictions. $\tau_{\mathrm{R}}=2.5 \times 10^{-5} \mathrm{~s}$ and $\tau_{\mathrm{E}}=0.089 \mathrm{~s}$. (b) Steady shear viscosity vs shear rate for $3.8 \mathrm{wt} \%$ HEUR22-4: experiments and model. $G_{\mathrm{m}}=3.8$.

more consistent with the previous observations of Sadgey and James ${ }^{26}$ in which they demonstrated the occurrence of slip when the shear stress exceeded roughly $500 \mathrm{~Pa}$. The critical shear stress for slip in our experiments can be estimated to be $200 \mathrm{~Pa}$ and is lower, as expected for a sample with stronger hydrophobic micelles.

4.3. Comparison with Extensional Rheology Experiments. Having demonstrated that the new model provides good agreement with the measured shear rheology of HEUR solutions over a range of concentrations, molecular weights, and endcap lengths, we now focus on the transient extensional rheology of these systems.

We use filament stretching rheometry ${ }^{63}$ to measure the tensile stress of extensional stresses in the same telechelic polymer solutions modeled above. We also explore the predictive power of the new two-species network constitutive model by comparing our measurements with numerical simulations of the startup of uniaxial extension without adjusting any of the constitutive parameters obtained from regression to steady shear flow.

A series of video frames for a 4 wt \% sample of HEUR22-2 stretched at an externally imposed strain rate of $\dot{E}=3.06 \mathrm{~s}^{-1}$ are shown in Figure 11. The images show the initial cylindrical liquid bridge configuration and its rapid extensional deformation into a necked configuration (as a result of the no-slip boundary

Figure 10. (a) Comparison of the linear viscoelastic response of 2.0 wt $\%$ HEUR23 with the model predictions. $\tau_{\mathrm{R}}=2.5 \times 10^{-5} \mathrm{~s}$ and $\tau_{\mathrm{E}}=4.85 \mathrm{~s}$. (b) Steady shear viscosity vs shear rate for $2.0 \mathrm{wt} \%$ HEUR23: experiments and model. $G_{\mathrm{m}}=7.8$.

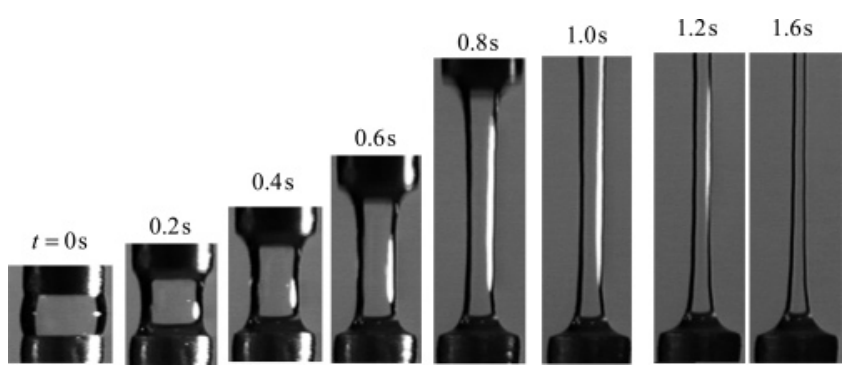

Figure 11. Images showing filament stretching of $4 \mathrm{wt} \%$ HEUR22-2 sample. The applied strain rate $\dot{E}=3.06 \mathrm{~s}^{-1}$. The diameter of the endplate is $3 \mathrm{~mm}$, and the initial sample length is $1.5 \mathrm{~mm}$.

condition at either endplate). The primary effect of nonNewtonian tensile stresses in the fluid is to establish an axially uniform cylindrical region over the central part of the filament. The diameter of the fluid thread subsequently decreases smoothly and monotonically with time over a large portion of the filament length, with two quasi-static fluid reservoirs connecting the fluid thread to the endplates. Because the filament does not evolve as an ideal cylinder, the kinematics at the midplane are not those expected from homogeneous uniaxial elongation, and the velocity profile must be corrected. ${ }^{63-65}$ The time evolution in the diameter is measured very close to the midplane using a laser micrometer, and this measurement is 
used to construct the corrected end plate displacement profile..$^{58}$ The subsequent experiment at the same strain rate can then be performed under conditions that generate ideal uniaxial elongation at the midplane (a so-called "type III" test ${ }^{65}$ ) in which the midplane diameter decreases in a smooth exponential manner that is well-described by $D_{\text {mid }}(t)=D_{0} \exp \left(-0.5 \dot{\epsilon}_{0} t\right)$.

Figure 12a shows the tensile force measurements of experiments performed using 4 wt \% solutions of HEUR22-2 and measured by the force transducer attached to the stationary lower plate (following correction for gravitational and surface tension terms ${ }^{66}$ ). In addition, the numerical predictions of the twospecies model in start-up of steady uniaxial elongational flow are shown for each sample. For each of these stretching experiments, the tensile force first grows rapidly at the onset of stretching, passes through a maximum that is dependent on strain rate, and then gradually decays as the filament diameter decays exponentially. These measurements can be combined with the measured diameter to evaluate the time evolution in the transient tensile stress difference $\Delta \tau(t)=4 F_{z}(t) / \pi D_{\text {mid }}^{2}(t)$, as shown in Figure 12b. In each case the time constants and the single nonlinear model parameter are identical to those values given in Figure 8c,d and are not adjusted to fit the measured data. The model appears to provide a good description of the extensional stress growth in startup of uniaxial extension of the HEUR22-2 fluid over a range of deformation rates, although it overpredicts the steady-state tensile stress at long times for the lowest deformation rate experiment. This might be in part due to some gravitational sagging in the column, which progressively invalidates the top-bottom symmetry assumed in the instrument operation and analysis. Similarly, the transient extensional viscosity for the 4 wt \% HEUR22-2 sample is found to be in good agreement with the model (except at small $\mathrm{De}$ and long times for the reason noted above). The extensional viscosity shows a transient response on time scales of order $\tau_{\mathrm{E}}$ followed by an approach to steady state with moderate extension rate thickening leading to Trouton ratios (scaled with a zero-shear rate viscosity of $\eta_{0}=63.6 \mathrm{~Pa} \cdot \mathrm{s}$ ) of $3 \leq \operatorname{Tr} \leq 10$.

Decreasing the concentration of HEUR polymer in solution results in a progressive decrease in the effective relaxation time and in the modulus of the micellar network; however, the two species network model still provides a good description of the evolution in the extensional stress difference as we show in Figure 12c for the 2 wt $\%$ HEUR22-2 solution.

As the concentration of HEUR polymer, and the associated network strength, is decreased, the Hencky strains that can be attained in homogeneous uniaxial extension are reduced due to the onset of a flow instability that is depicted in the sequence of high-speed digital video images shown in Figure 13a. A defect appears in the filament (typically close to the axial midplane), and a tear rapidly propagates radially across the fluid thread. Because the fluid is in tension, the scission into two topologically distinct domains is enhanced by elastic recoil toward the two end plates. The two blobs undergo a series of inertio-elastic damped oscillations that decay over time scales longer than this image sequence. The tear surface present for short times following the rupture event is shown quite clearly in the last three frames of Figure 13a. Similar rupture instabilities are observed in polymer melts when the tensile stresses in the entangled network result in catastrophic loss of entanglements. ${ }^{67}$ In the present system, the imposed elongational flow results in disruption of the network of interconnected flower micelles and a progressive collapse in the tensile stress. Viscoelastic necking and rupture instabilities are commonly described phenomeno-
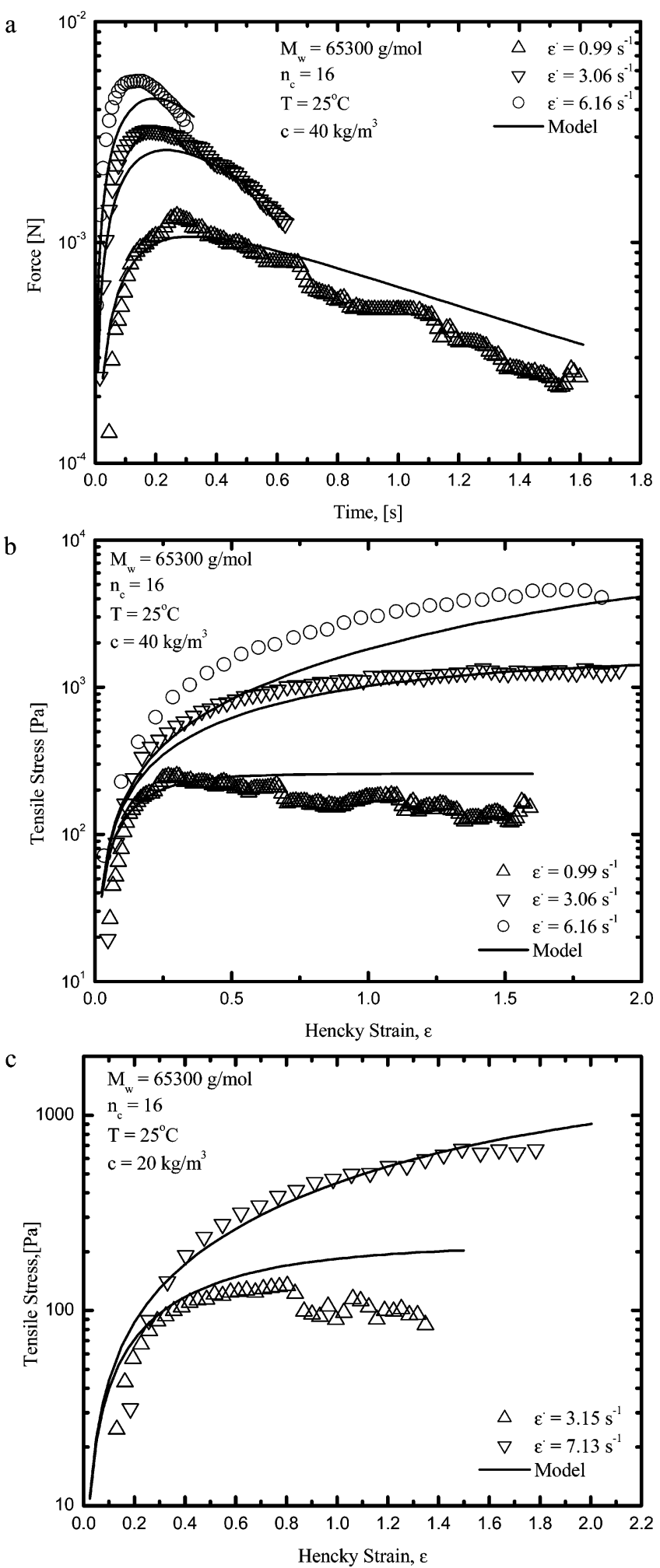

Figure 12. (a) Transient tensile force as a function of time for $4 \mathrm{wt} \%$ HEUR22-2: experiments and model predictions. (b) Transient extensional stress as a function of Hencky strain for 4 wt \% HEUR22-2: experiments and model predictions. (c) Transient extensional stress as a function of Hencky strain for 2 wt \% HEUR22-2: experiments and model predictions.

logically by the Considère criterion, which states that homogeneous uniaxial elongation in an elastic material cannot be maintained beyond the strain at which the tensile force passes through a maximum. ${ }^{68}$ We denote this critical strain as the "failure strain" $\epsilon_{\mathrm{f}}$. However, this stability criterion does not provide any information on the subsequent time required for 

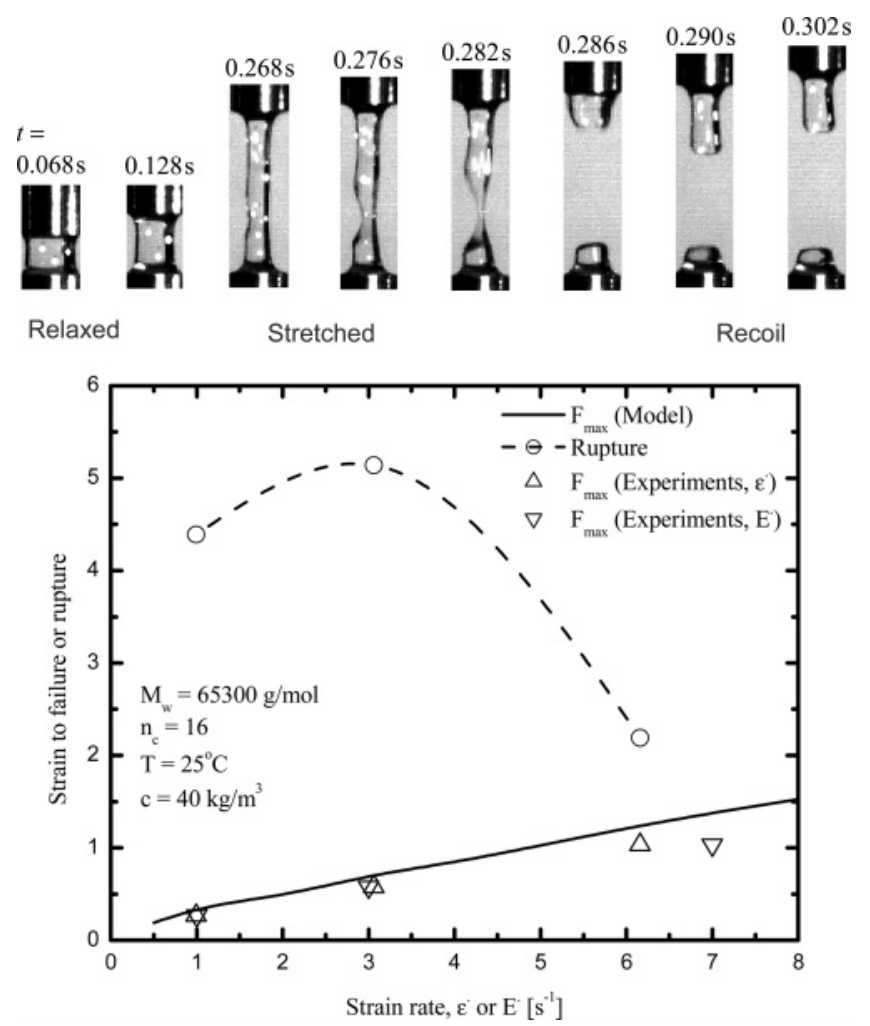

Figure 13. (a, top) Images showing rupture of $2.4 \mathrm{wt} \%$ HEUR22-2. The applied strain rate is $\dot{E}=3 \mathrm{~s}^{-1}$. (b, bottom) Strain to failure and strain to rupture during the extension of $4 \mathrm{wt} \%$ HEUR22-2 filament.

the instability to propagate completely across the elongating sample and lead to filament rupture. In an elastic solid this rupture may be very rapid, whereas in viscoelastic fluids the defect propagation will depend on the specific details of the fluid rheology and constitutive model. ${ }^{69,70}$

In Figure 13b we show the results of filament stretching experiments at three different strain rates and plot the strains at which the force passes through a maximum (together with the model predictions) and also the value of the strain (determined from high-speed video images at 500 frames/s) at which the sample completely bifurcates into two domains. Although the constitutive model accurately describes the strain at which the force passes through a maximum, it is clear that the time required for instability growth and complete sample failure can be quite long at low strain rates. As the imposed strain rate increases and the effective Deborah number grows, the two curves approach each other, in agreement with general theoretical expectations of approach toward a rapid stretching limit in which viscous effects become negligible. ${ }^{68,69}$

Finally, in Figure 14 we show the measured steady-state elongational viscosity together with the predictions of the new network model as a function of the imposed strain rate. The HEUR solutions show tension thickening at intermediate $D e$, and this is predicted to be followed by rate thinning at high Deborah numbers; however, our present filament stretching device is unable to achieve deformation rates beyond $10 \mathrm{~s}^{-1}$. For consistency with our initial theoretical model considerations, we scale the elongation rate with the natural intrinsic time scale of the model $\tau_{\mathrm{E}}$. For this reason, appreciable elastic effects appear to develop at Deborah numbers less than 0.5; however, the effective network time scale $\tau_{\text {eff }}$ may be significantly longer ( see eq 5 or 31 ). ${ }^{36}$ For comparison, we also show by the dashed lines the sensitivity of the model predictions to changes in the single nonlinear model parameter, $G_{\mathrm{m}}$. Although changing this

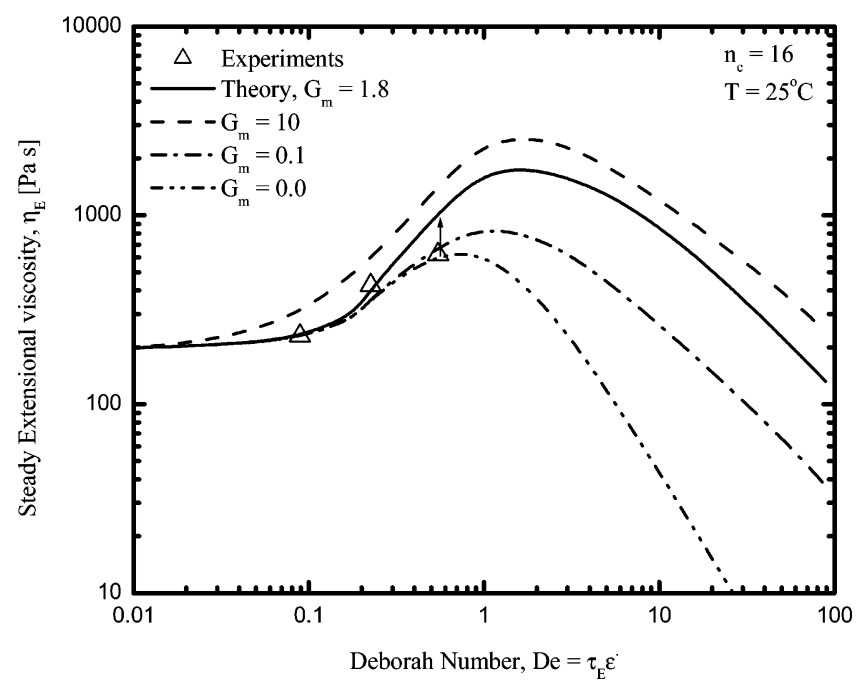

Figure 14. Steady extensional viscosity as a function of Deborah number for $4 \mathrm{wt} \%$ HEUR22-2: experiments and model predictions for different $G_{\mathrm{m}}$.

parameter by an order of magnitude affects the quantitative model predictions, it does not change the qualitative trends observed in the figure for finite values of $G_{\mathrm{m}}$. When the model parameter is set to $G_{\mathrm{m}}=0$, the model predicts much more rapid rate-thinning at high Deborah numbers. This suggests that, in the absence of any strain-induced incorporation of hydrophobes, the number of active chains decreases very promptly under uniaxial extension.

The dynamics of necking instabilities in viscoelastic fluid threads have been reviewed recently by Renardy, ${ }^{71}$ and it is noted that fluid threads described by constitutive models in which the extensional viscosity passes through a maximum may undergo a purely elastic mode of necking failure in which surface tension plays no role. Our observations of network rupture shown in Figure 13 appear to be consistent with this expectation.

\section{Conclusions}

In this paper we have reported an experimental and theoretical investigation of the nonlinear rheological properties of telechelic associative polymers. Shear and extensional flow experiments were performed using a series of model hydrophobically modified ethoxylate-urethane (HEUR) polymers with varying degrees of hydrophobicity. Initial attempts were made to describe these experimental observations using the VaccaroMarrucci model, ${ }^{40}$ but only qualitative agreement could be attained. Similar conclusions were reached by Pellens et al..$^{3,4}$ On the basis of insights from Brownian dynamics simulations by van den Brule and co-workers, ${ }^{38,39}$ a new closed-form constitutive model-which incorporates important molecular features of the associative polymer solutions - has been developed to describe the observed nonlinear flow properties. The model incorporates contributions to the total stress tensor from both the "elastically active" bridging chains between micelles and the dangling chains that continuously exit and reenter the micellar junctions. Nonlinear chain extension, the shear-induced enhancement of associations, and the stretch-induced dissociation of hydrophobic chains are essential features of the model. The resulting constitutive equation is summarized in Table 2, and closed form expressions for the linear viscoelastic properties, the zero-shear rate viscosity, and first normal stress difference are derived in section 3 . Without the use of adjustable functions the model accurately predicts the experimentally observed 
power-law dependence of zero-shear-rate viscosity on polymer concentration; $\eta_{0} \sim c^{2.75}$. Telechelic polymer systems commonly exhibit dual relaxation behavior arising from the Rouse relaxation time $\tau_{\mathrm{R}}$ of the individual polymer chains $\left(\sim 5 \times 10^{-5} \mathrm{~s}\right)$ and from the "effective" network relaxation time $\tau_{\text {eff }}\left(\sim 10^{-1}\right.$ s). The model predicts that this "effective" network relaxation time depends on the concentration and molecular weight of the chains, the length of the hydrophobic end groups, and the aggregation number of the micelles and is of the form $\tau_{\text {eff }}=(\chi \Omega)^{-1}\left(3 N_{\mathrm{agg}} M_{\mathrm{w}} / 8 \pi c N_{\mathrm{A}}\right)^{-2 / 3} \mathrm{e}^{0.98 n_{\mathrm{c}}}$. This agrees with the nonlinear dependence on concentration $\tau_{\text {eff }} \sim c^{2 / 3}$ observed experimentally.

The model and the experimental measurements both show a marked deviation from the Cox-Merz rule. The measured steady shear viscosity $\eta(\dot{\gamma})$ for a number of the HEUR samples show considerable shear thickening, prior to the onset of shear thinning at higher shear rates. These features are captured by the new model using a single dimensionless constitutive parameter $G_{\mathrm{m}}$, which describes the orientational and deformation-rate-dependent creation rate of the active chains. The relative magnitude of this parameter with respect to the nonlinear stretching of the elastically active chains also allows us to describe quantitatively the observed shift in the onset of shear thickening and subsequent shear thinning toward lower shear rates upon an increase in polymer concentration or molecular weight. Computed profiles of the first normal stress difference are monotonic and do not show a local maximum, indicating that, in this model at least, the shear thinning at large deformation rates is a result of progressive network deformation rather than network collapse.

We also report the first quantitative comparisons of model predictions for the transient extensional rheological properties with experimental measurements obtained using a uniaxial filament stretching device. Using the same values of the constitutive parameter $G_{\mathrm{m}}$ determined from steady flow, the model predicts extensional stress growth profiles that are in close agreement with data. The results show a moderate strain hardening in the transient extensional viscosity of HEUR polymer solutions at intermediate strains, and the transient extensional stress difference approaches a steady state for Hencky strains greater than two. The elongating samples ultimately undergo a viscoelastic rupture event. The filament necking and rupture processes observed experimentally appear to be connected to an instability resulting from saturation in the tensile stresses and a local maximum in the steady-state extensional viscosity at a critical deformation rate.

The new two-species model is shown to quantitatively capture almost all of the nonlinear features that have been observed in telechelic associative polymers. However, additional refinement of the model is needed to investigate several unexplored properties such as temperature dependence of the rheological functions, the effect of looped chains (whose contributions were assumed to be negligible), and the consequence of closure approximations. The dependence of the network relaxation time on temperature contains an Arrhenian contribution from the jump rate $\tau_{\mathrm{E}}{ }^{-1}$ but also additional contributions because of the unknown nonlinear dependence of the aggregation number $N_{\text {agg }}$ in associative polymer systems on temperature. ${ }^{8,9,15}$ At very high concentrations of HEUR polymers, the additional presence of looped chains is likely to generate repulsive potentials ${ }^{10}$ which will change the creation and destruction rates of the elastically active chains. The quality of the closure approximations employed in deriving closed forms for the network creation and destruction terms, as well as the orientational dependence of the creation rate, can be best explored using Brownian dynamics or other microscale simulation methods.

The recent success of closed-form two-species network models such as the present one in describing the linear and nonlinear rheological properties of telechelic polymers suggests that such models may also be useful (through the incorporation of appropriate network creation and destruction terms) in modeling other complex fluids featuring temporary physical networks. Such systems include hydrophobically modified alkali-soluble ethoxylates (HASE) and wormlike surfactant micellar systems.

Acknowledgment. We are grateful to Dr. Richard Jenkins for providing us with the HEUR polymers. K.C.T. thanks Nanyang Technological University for the financial support during his sabbatical leave at MIT.

\section{References and Notes}

(1) Winnik, M. A.; Yekta, A. Curr. Opin. Colloid Interface Sci. 1997, 2, 424-436.

(2) Larson, R. G. The Structure and Rheology of Complex Fluids; Oxford University Press: New York, 1999.

(3) Pellens, L.; Corrales, R. G.; Mewis, J. J. Rheol. 2004, 48, 379-393.

(4) Pellens, L.; Ahn, K. H.; Lee, S. J.; Mewis, J. J. Non-Newtonian Fluid Mech. 2004, 121, 87-100.

(5) Annable, T.; Buscall, R.; Ettelaie, R.; Whittlestone, D. J. Rheol. 1993 $37,695-726$

(6) Lafleche, F.; Durand, D.; Nicolai, T. Macromolecules 2003, 36, 13311340.

(7) Lafleche, F.; Nicolai, T.; Durand, D.; Gnanou, Y.; Taton, D. Macromolecules 2003, 36, 1341-1348.

(8) Pham, Q. T.; Russel, W. B.; Thibeault, J. C.; Lau, W. Macromolecules 1999, 32, 5139-5146

(9) Pham, Q. T.; Russel, W. B.; Thibeault, J. C.; Lau, W. Macromolecules 1999, 32, 2996-3005.

(10) Semenov, A. N.; Joanny, J. F.; Khokhlov, A. R. Macromolecules 1995, $28,1066-1075$.

(11) Annable, T.; Buscall, R.; Ettelaie, R. Colloids Surf., A 1996, 112, 97116.

(12) Jenkins, R. D. The Fundamental Thickening Mechanism of Associative Polymers in Latex Systems. Ph.D., Lehigh University, Bethlehem, 1991.

(13) Witten, T. A. J. Phys. (Paris) 1988, 49, 1055-1063

(14) Xu, B.; Yekta, A.; Li, L.; Masoumi, Z.; Winnik, M. A. Colloids Surf., A 1996, 112, 239-250.

(15) Yekta, A.; Xu, B.; Duhamel, J.; Adiwidjaja, H.; Winnik, M. A. Macromolecules 1995, 28, 956-966.

(16) Yekta, A.; Duhamel, J.; Adiwidjaja, H.; Brochard, P.; Winnik, M. A. Langmuir 1993, 9, 881-883.

(17) Lundberg, R. D.; Duvdevani, I. ACS Symp. Ser. 1991, 462, 155-175

(18) Tam, K. C.; Jenkins, R. D.; Winnik, M. A.; Bassett, D. R. Macromolecules 1998, 31, 4149-4159.

(19) Duhamel, J.; Yekta, A.; Hu, Y. Z.; Winnik, M. A. Macromolecules 1992, 25, 7024-7030

(20) Ng, W. K.; Tam, K. C.; Jenkins, R. D. J. Rheol. 2000, 44, 137-147.

(21) Ma, S. O. X.; Cooper, S. L. Macromolecules 2001, 34, 3294-3301.

(22) Aubry, T.; Moan, M. J. Rheol. 1994, 38, 1681-1692.

(23) Serero, Y.; Jacobsen, V.; Berret, J. F.; May, R. Macromolecules 2000, 33, 1841-1847.

(24) Berret, J. F.; Sereo, Y.; Winkelman, B.; Calvet, D.; Collet, A.; Viguier, M. J. Rheol. 2001, 45, 477-492.

(25) Berret, J. F.; Serero, Y. Phys. Rev. Lett. 2001, 8704, art. no.-048303.

(26) Sadeghy, K.; James, D. F. J. Non-Newtonian Fluid Mech. 2000, 90, $127-158$.

(27) Tan, H.; Tam, K. C.; Tirtaatmadja, V.; Jenkins, R. D.; Bassett, D. R. J. Non-Newtonian Fluid Mech. 2000, 92, 167-185.

(28) Tanaka, F.; Edwards, S. F. J. Non-Newtonian Fluid Mech. 1992, 43, 247-271.

(29) Tanaka, F.; Edwards, S. F. J. Non-Newtonian Fluid Mech. 1992, 43, $273-288$.

(30) Tanaka, F.; Edwards, S. F. J. Non-Newtonian Fluid Mech. 1992, 43, 289-309.

(31) Tanaka, F.; Edwards, S. F. Macromolecules 1992, 25, 1516-1523.

(32) Green, M. S.; Tobolsky, A. J. Chem. Phys. 1946, 14, 80.

(33) Yamamoto, M. J. J. Phys. Soc. Jpn. 1958, 13, 1200.

(34) Wang, S. Q. Macromolecules 1992, 25, 7003-7010.

(35) Witten, T. A.; Cohen, M. H. Macromolecules 1985, 18, 1915. 
(36) Marrucci, G.; Bhargava, S.; Cooper, S. L. Macromolecules 1993, 26 , 6483-6488.

(37) Ahn, K. H.; Osaki, K. J. Non-Newtonian Fluid Mech. 1995, 56, 267288.

(38) Vandenbrule, B. H. A. A.; Hoogerbrugge, P. J. J. Non-Newtonian Fluid Mech. 1995, 60, 303-334.

(39) Hernandez-Cifre, J. G.; Barenbrug, T. M. A. O. M.; Schieber, J. D.; van den Brule, B. H. A. A. J. Non-Newtonian Fluid Mech. 2003, 113, 73-96.

(40) Vaccaro, A.; Marrucci, G. J. Non-Newtonian Fluid Mech. 2000, 92 261-273.

(41) Bell, G. I. Science 1978, 178, 618.

(42) Bird, R. B.; Curtiss, C. F.; Armstrong, R. C.; Hassager, O. Dynamics of Polymeric Fluids; Wiley: New York, 1987; Vol. 2.

(43) Ottinger, H. C.; Petrillo, F. J. Rheol. 1996, 40, 857-874.

(44) Tanner, R. I. Engineering Rheology; Oxford University Press: New York, 1985.

(45) Peterlin, A. Makromol Chem. 1961, 44, 338

(46) Doyle, P. S.; Shaqfeh, E. S. G.; McKinley, G. H.; Spiegelberg, S. H. J. Non-Newtonian Fluid Mech. 1998, 76, 79-110.

47) Keunings, R. J. Non-Newtonian Fluid Mech. 1997, 68, 85-100.

(48) Petruccione, F.; Biller, P. J. Chem. Phys. 1988, 89, 577-582.

(49) Hatzikiriakos, S. G.; Vlassopoulos, D. Rheol. Acta 1996, 35, 274287.

(50) Evans, E.; Ritchie, K. Biophys. J. 1997, 72, 1541-1555.

(51) Daoud, M.; Cotton, J. P. J. Phys. (Paris) 1982, 43, 531

(52) Haber, C.; Wirtz, D. Biophys. J. 2000, 79, 1530-1536.

(53) Boltenhagen, P.; Hu, Y. T.; Matthys, E. F.; Pine, D. J. Europhys. Lett. 1997, 38, 389-394.

(54) Zipfel, J.; Lindner, P.; Tsianou, M.; Alexandridis, P.; Richtering, W. Langmuir 1999, 15, 2599-2602.

(55) Richey, B.; Kirk, A. B.; Eisenhart, E. K.; Fitzwater, S.; Hook, J. J. Coat.Technol. 1991, 63, 31-40.
(56) Jenkins, R. D.; Bassett, D. R.; Silebi, C. A.; Elaasser, M. S. J. Appl. Polym. Sci. 1995, 58, 209-230.

(57) Anna, S. L.; McKinley, G. H. J. Rheol. 2001, 45, 115-138.

(58) Anna, S. L.; Rogers, C.; McKinley, G. H. J. Non-Newtonian Fluid Mech. 1999, 87, 307-335.

(59) Anna, S. L.; McKinley, G. H.; Nguyen, D. A.; Sridhar, T.; Muller, S J.; Huang, J.; James, D. F. J. Rheol. 2001, 45, 83-114.

(60) Graessley, W. W. Polymer 1980, 21, 258.

(61) Donth, E.-J. Relaxation and Thermodynamics in Polymers: Glass Transition; John Wiley: New York, 1992.

(62) Le Meins, J. F.; Tassin, J. F. Macromolecules 2001, 34, 2641-2647.

(63) McKinley, G. H.; Sridhar, T. Annu. Rev. Fluid Mech. 2002, 34, 375415 .

(64) Orr, N. V.; Sridhar, T. J. Non-Newtonian Fluid Mech. 1999, 82, 203232.

(65) Kolte, M. I.; Rasmussen, H. K.; Hassager, O. Rheol. Acta 1997, 36 , $285-302$.

(66) Szabo, P.; McKinley, G. H. Rheol. Acta 2003, 42, 269-272.

(67) Joshi, Y. M.; Denn, M. M. J. Rheol. 2004, 48, 591-598.

(68) Malkin, A. Y.; Petrie, C. J. S. J. Rheol. 1997, 41, 1-25.

(69) Hassager, O.; Kolte, M. I.; Renardy, M. J. Non-Newtonian Fluid Mech. 1998, 76, 137-151.

(70) Yao, M. W.; McKinley, G. H.; Debbaut, B. J. Non-Newtonian Fluid Mech. 1998, 79, 469-501.

(71) Renardy, M. Self-Similar Breakup of Non-Newtonian Fluid Jets; In Rheology Reviews; The British Society of Rheology: 2004; Vol. 2 pp 171-196.

MA051614X 